\title{
Protective Zika vaccines engineered to eliminate enhancement of dengue infection via immunodominance switch
}

\author{
Lianpan Dai $\circledast^{1,2,3,8} \bowtie$, Kun $\mathrm{Xu}^{3,8}$, Jinhe Li ${ }^{2,4,8}$, Qingrui Huang ${ }^{5}$, Jian Song ${ }^{1}{ }^{1}$, Yuxuan $\mathrm{Han}^{2}$, \\ Tianyi Zheng ${ }^{2,4}$, Ping Gao ${ }^{2,4}$, Xuancheng Lu ${ }^{6}$, Huabing Yang ${ }^{(5}{ }^{5}$, Kefang Liu', Qianfeng $\mathrm{Xia}^{3}$, \\ Qihui Wang ${ }^{1}$ ', Yan Chai', Jianxun Qi ${ }^{1}$ ', Jinghua Yan $\oplus^{5} \bowtie$ and George F. Gao $\oplus^{1,2,4 凶}$
}

\begin{abstract}
Antibody-dependent enhancement (ADE) is an important safety concern for vaccine development against dengue virus (DENV) and its antigenically related Zika virus (ZIKV) because vaccine may prime deleterious antibodies to enhance natural infections. Cross-reactive antibodies targeting the conserved fusion loop epitope (FLE) are known as the main sources of ADE. We design ZIKV immunogens engineered to change the FLE conformation but preserve neutralizing epitopes. Single vaccination conferred sterilizing immunity against ZIKV without ADE of DENV-serotype 1-4 infections and abrogated maternal-neonatal transmission in mice. Unlike the wild-type-based vaccine inducing predominately cross-reactive ADE-prone antibodies, B cell profiling revealed that the engineered vaccines switched immunodominance to dispersed patterns without DENV enhancement. The crystal structure of the engineered immunogen showed the dimeric conformation of the envelope protein with FLE disruption. We provide vaccine candidates that will prevent both ZIKV infection and infection-/vaccination-induced DENV ADE.
\end{abstract}

Z IKV is a mosquito-borne pathogen belonging to the genus Flavivirus in the family Flaviviridae ${ }^{1}$. The 2015-2016 ZIKV epidemic spread to 84 countries worldwide, including China $^{2,3}$. ZIKV infection during pregnancy can lead to miscarriage and catastrophic congenital Zika syndrome in neonates, including microcephaly and neurodevelopmental disorders ${ }^{4-6}$, and is also associated with Guillain-Barré syndrome in adults ${ }^{7}$. Evidence so far has demonstrated that ZIKV can break through barriers from blood to brain, placenta, testes and eyes to cause pathogenicity ${ }^{8}$. We and others found that ZIKV can cause testis damage and male infertility in a mouse model ${ }^{9,10}$. Nevertheless, no prophylactics or therapeutics are available, highlighting the need for vaccine development. With global efforts to develop vaccines, a notable concern has been raised that ZIKV vaccine may prime cross-reactive antibodies to enhance natural infections of the antigenically related DENV via $\mathrm{ADE}^{11-14}$. Antibodies that can bind flavivirus particles without neutralizing activities will cause ADE via Fcy receptor-mediated virus uptake $^{14,15}$. Extensive ADE was observed in heterotypic secondary DENV infection and can explain severe dengue disease in human ${ }^{16}$. $\mathrm{ADE}$ has been the principal hurdle to DENV vaccine development and is implicated as a limitation of the first licensed dengue vaccine, Dengvaxia, which is recommended only for DENV-seropositive, not DENV-seronegative, individuals due to more frequent severe clinical outcomes ${ }^{11,14,17,18}$. ADE also complicated ZIKV vaccine development itself. Mounting evidence has demonstrated that pre-existing ZIKV antibodies significantly enhance DENV infection in vitro and aggravated dengue disease in vivo using both mouse and macaque models ${ }^{12,13,19,20}$. More importantly, a recent study of pediatric cohorts in Nicaragua clearly shows that previous ZIKV infection enhanced future risk of DENV2 disease and severity, as well as DENV3 severity, in humans ${ }^{21,22}$. This highlights that an effective and safe ZIKV vaccine is highly needed to (1) prevent homogenous ZIKV infection; (2) prevent future severe DENV infection caused by natural ZIKV infection and (3) prevent vaccine-induced DENV ADE. In particular, ZIKV infection was found to activate ADE-prone antibodies de novo in DENV-experienced individuals ${ }^{23}$. Therefore, the possible risk for DENV ADE should be taken into account for ZIKV vaccine design, especially, given that ZIKV/DENV are transmitted by same vectors (usually Aedes aegypti) and endanger people living in same regions ${ }^{11}$.

ADE-prone antibodies target mainly epitopes on precursor membrane protein (prM) and fusion loop (FL) of envelope protein $(\mathrm{E})^{24,25}$ in DENV. These antibodies are cross-reactive between serotypes or serocomplexes of flavivirus due to epitope conservation, and are usually poorly neutralizing ${ }^{26}$. More potent neutralizing antibodies (NAbs) recognize other epitopes ${ }^{26-30}$. For ZIKV, neutralizing monoclonal antibodies (mAbs) have been reported to recognize epitopes on domains I (DI), DII and DIII, or quaternary epitopes on the virus surface $e^{1,27,2,28,31,32}$. Strategies to prevent induction of ADE-prone antibodies have been described recently for the design of new ZIKV vaccines ${ }^{1,13}$. Either mutation ${ }^{13}$ or minimal exposure ${ }^{11}$ of the FL epitope (FLE) was demonstrated to reduce the ADE of DENV infection in mice. However, after ZIKV challenge, substantial virus burden can still be detected in sera ${ }^{13}$, or in targeted tissues

'CAS Key Laboratory of Pathogen Microbiology and Immunology, Institute of Microbiology, Chinese Academy of Sciences (CAS), Beijing, China. ${ }^{2}$ University of Chinese Academy of Sciences, Beijing, China. ${ }^{3}$ Key Laboratory of Tropical Translational Medicine of Ministry of Education, School of Tropical Medicine and Laboratory Medicine, The First Affiliated Hospital, Hainan Medical University, Hainan, China. ${ }^{4}$ Research Network of Immunity and Health (RNIH), Beijing Institutes of Life Science, Chinese Academy of Sciences, Beijing, China. ${ }^{5}$ CAS Key Laboratory of Microbial Physiological and Metabolic Engineering, Institute of Microbiology, Chinese Academy of Sciences, Beijing, China. ${ }^{6}$ Laboratory Animal Center, Chinese Center for Disease Control and Prevention (China CDC), Beijing, China. ${ }^{7}$ Faculty of Health Sciences, University of Macau, Macau SAR, China. ${ }^{8}$ These authors contributed equally: Lianpan Dai, Kun Xu, Jinhe Li. $\bowtie$ e-mail: dailp@im.ac.cn; yanjh@im.ac.cn; gaof@im.ac.cn 
including spleen, brain, placenta and fetal head ${ }^{11,13}$. Given the many detrimental effects observed on fetuses and neonates following ZIKV infection in pregnant women, sterilizing immunity may be required for vaccine development to prevent long-term sequelae $e^{33}$.

Here, we described an antibody-to-vaccine development process. By extracting insights from the structural basis of FLE mAbs, we engineered a ZIKV immunogen to (1) eliminate the FLE while (2) still presenting potent neutralizing epitopes. Introducing mutations to key residues of FLE could easily cause immunogen impairment, and this obstacle was overcome by homologous replacement by naturally occurring variants from arthropod-specific flaviviruses. The resultant immunogens, MutB/C, were developed as ZIKV vaccines. Impressively, a single vaccination was completely protective and sterilizing, with elimination of ADE for DENV in mice, and prevented maternal-fetal transmission against ZIKV challenge. By analysis of vaccine-induced B cell pools at single-cell level, we found that, unlike the wild-type (WT) construct inducing predominantly cross-reactive ADE-prone antibodies, MutB/C-based vaccines switched immunodominance to ZIKV-specific patterns without DENV enhancement. The crystal structure of the immunogen further revealed the molecular basis for the vaccine potency and ADE abrogation.

\section{Results}

ZIKV vaccines expressing either prM/E or M/E induce DENV ADE. We previously described a protective ZIKV vaccine using chimpanzee adenovirus type 7 (AdC7) as a vector to express $\mathrm{ZIKV}-\mathrm{M} / \mathrm{E}^{34}$. Since many of the developing $\mathrm{ZIKV}$ vaccines are based on $\mathrm{prM} / \mathrm{E}^{33}$, we also generated $\mathrm{AdC}$ expressing prM/E for comparison. These two vaccines were given to groups of BALB $/ \mathrm{c}$ mice. Four weeks later, sera were first assessed for neutralizing activities against ZIKV infection, showing comparable magnitudes of neutralizing titers between these two groups (Extended Data Fig. 1a). Next, sera were evaluated for their ability to enhance DENV infection on K562 cells bearing the $\mathrm{F} c \gamma$ receptor IIA (CD32A). Notably, dilutions of sera from mice immunized with either AdC7-M/E or AdC7-prM/E dramatically enhanced the infections of all four serotypes of DENV, implicating potential safety concerns (Extended Data Fig. 1b-e).

Rational design of ZIKV immunogens. We previously isolated a group of FLE mAbs from a ZIKV-infected patient ${ }^{31}$. A representative mAb, Z6, was evaluated for ADE of DENV infection. As expected, Z6 promoted all four serotypes of DENV infections as bell-shaped curves (Extended Data Fig. 1f). To better understand the FLE to guide vaccine design, we determined the crystal structure of Z6 bound to ZIKV-E at a resolution of $3.0 \AA$ (Supplementary Table 1) as a glimpse at the first structure of a human FLE mAb. Z6 straddled FL from the top of DII with balanced interactions with both heavy and light chains (Fig. 1a,b and Extended Data Fig. 2a,e). Comparing the three structures of known FLE mAbs (Z6, 2A10G6 and $\mathrm{E} 53)^{35,36}$, we found they approached FL with different angles, contacting residues within W101-K110 with the help from the $b c$ loop (Fig. 1a,b and Extended Data Fig. 2). The structural analyses pinpoint potential residues for engineering to destroy the FLE.

Next, we sought to engineer ZIKV immunogen. M/E was chosen as template because (1) it was previously demonstrated as a protective immunogen ${ }^{34,37,38}$, and (2) it avoided the precursor peptideanother main target for ADE-prone antibodies ${ }^{24,25,39}$. To characterize the immunogens antigenically, mAbs bound to different regions of ZIKV-E were used as probes. Z6 and 2A10G6 bound to FLE, whereas Z3L1, Z20 and Z23 were ZIKV-specific neutralizing mAbs that bound epitopes on DI, DII and DIII, respectively ${ }^{31}$ (Fig. 1c).

Given that residue W101 in FL was demonstrated previously to be bound by most FLE antibodies ${ }^{26,40}$ and contributed most interactions to Z6 and 2A10G6 (Fig. 1b and Extended Data Fig. 2e), we therefore attempted to mutate W101 to reduce FLE. As expected, W101 mutated to the other 19 amino acids, except W101F, abrogated the interactions with FLE mAbs (Extended Data Fig. 3a,b). Nevertheless, these mutations dramatically reduced antigen expression and epitope stability. In particular, the DI/DII epitopes for Z3L1 and Z20 were barely probed (Extended Data Fig. 3b), implying a sacrifice of immunogenicity. Therefore, we investigated other strategies.

Structural analysis showed that, except for W101, other FL residues, including G106, L107 and W108 (Fig. 1b and Extended Data Fig. 2) are also important for FLE. We therefore sought to replace the entire FL. FL amino acids are highly conserved within the genus Flavivirus, but have variations in distantly related species such as arthropod-specific flaviviruses (Fig. 1d and Extended Data Fig. 4a). We assumed these naturally occurring FL variants may be structurally stable due to evolutionary selection. Therefore, we substituted FL residues (D98-G109) of ZIKV with their homologs in arthropod-specific flaviviruses, namely MutA, MutB and MutC (Fig. 1d). Five mutations were introduced in each construct (D98N, N103T, G106F/L, L107E/K and F108W) (Fig. 1d). These mutants exhibited no discernable binding to FLE mAbs, yet substantial antigen expression displaying neutralizing epitopes probed by Z3L1, Z23 and Z20 (Fig. 1e). Antigen expression can also be detected in supernatant from transfected cells by capture enzyme-linked immunosorbent assay (ELISA) (Fig. 1f). Due to the relatively low antigen expression of MutA (Fig. 1e,f), we focused on the other two designs, MutB and MutC, for further immunogen evaluation.

We generated AdC7-M/E-MutB and AdC7-M/E-MutC as vaccines. Both vaccines had substantial antigen expression of correctly folded both intracellular and secreted antigen when infecting cells (Extended Data Fig. 5). We immunized groups of BALB/c mice with AdC7-M/E-MutB or AdC7-M/E-MutC. AdC7-M/E-WT and PBS were given as controls; 4 weeks later, mice sera were collected for measurement. We observed comparable ZIKV-E-reactive serologic responses between groups of mice receiving vaccines based on WT, MutB or MutC, respectively (Fig. 1g). Moreover, sera from the WT group were highly cross-reactive to four serotypes of DENV; however, sera from $\mathrm{MutB} / \mathrm{C}$ groups showed little or no cross-reactions to DENV1-4 (similar to sham) (Fig. 1g).

Fig. 1 | Engineering ZIKV immunogens. a, Crystal structure of Z6-Fab bound to ZIKV-sE. b, Residues participating in interactions between ZIKV-sE and Z6-Fab. The numbers of contacts are highlighted for the main contributors in ZIKV-sE, and these residues are labeled in a. c, ZIKV-E-dimer structure shown as surface. The schematic binding sites for the indicated $\mathrm{mAbs}$ are highlighted in ellipses. $\mathbf{d}$, Sequence alignment of FL regions from members of flaviviruses. The variant residues in FL of arthropod-specific flaviviruses are highlighted in different colors. Designs to substitute ZIKV-E FL with homologs from three types of arthropod-specific flaviviruses are termed MutA, MutB and MutC, respectively, represented by homologous sequences from Aedes flavivirus, cell fusing agent virus and Nakiwogo virus, respectively. e, Flow cytometry detection of antigen expression and epitope display probed by FLE mAbs or neutralizing mAbs targeting epitopes on DI, DII or DIII. f, Capture ELISA quantifying the secretion of ZIKV antigen from transfected cells. Data are means of triplicates. $\mathbf{g}$, Female BALB/c mice $\left(n=6 ; 6-8\right.$ weeks old) were immunized with a single dose $\left(1.6 \times 10^{11} \mathrm{vp}\right)$ of AdC7-M/E-WT, AdC7-M/E-MutB or AdC-M/E-MutC via the i.m. route. PBS was given as the sham vaccine. Serum samples were collected at 4 weeks postimmunization. ELISA analyses show antibody titers to ZIKV-E or cross-binding to DENV1-E, DENV2-E, DENV3-E and DENV4-E, respectively. Data are means \pm s.e.m. $P$ values were analyzed with one-way ANOVA (NS, not significant; ${ }^{\star} P<0.05,{ }^{\star \star \star \star} P<0.0001 ; P$ values are available in source data). The dashed line indicates $L O D$. 
MutB/C vaccines induced protective immunity without DENV ADE. To evaluate vaccine efficacy, serum samples were further assessed for neutralizing activities against ZIKV infection (SMGC-1 strain). Impressively, MutB/C-based vaccines elicited magnitudes of neutralizing titers comparable to those elicited by the WT form (Fig. 2a). High levels of ZIKV-E-binding antibodies and NAbs were detected 12- and 24-weeks postvaccination until our last serum sam- pling (Extended Data Fig. 4b,c). To further explore the prophylactic efficacy of FL-engineered vaccines, IFNAR1-deficient (Ifnar1 ${ }^{-/-}$) mice were used as a sensitive model for ZIKV challenge ${ }^{34,41}$. At 4 weeks after a single-dose immunization, mice receiving either the WT- or MutB/C-based vaccines developed high levels of ZIKV NAbs (Extended Data Fig. 4d). At day 30 postvaccination, they were challenged with a lethal dose $\left(5 \times 10^{6}\right.$ focus-forming units (FFU) a

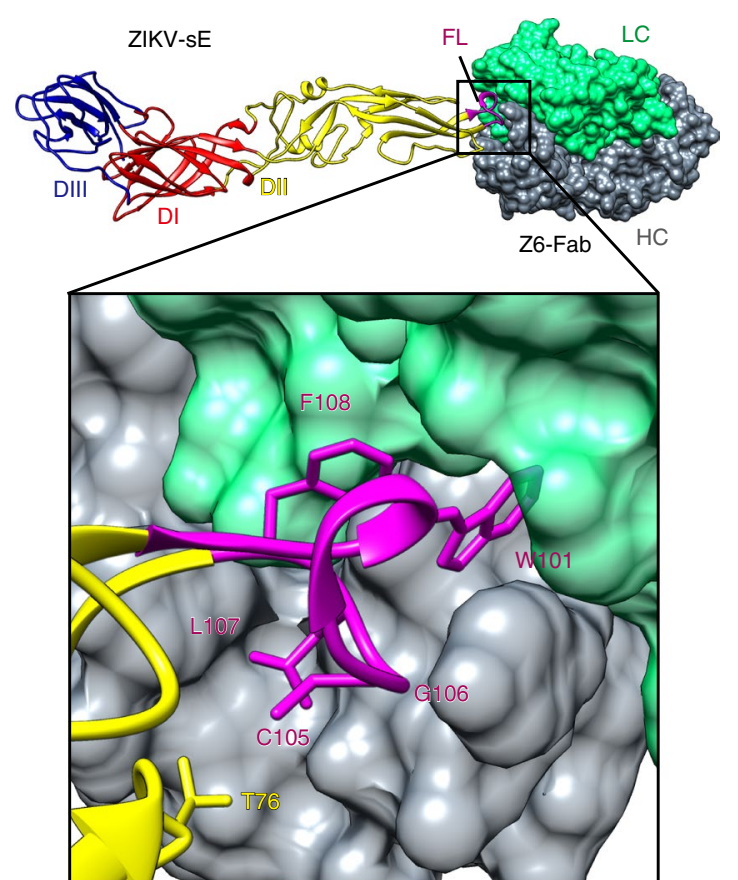

c

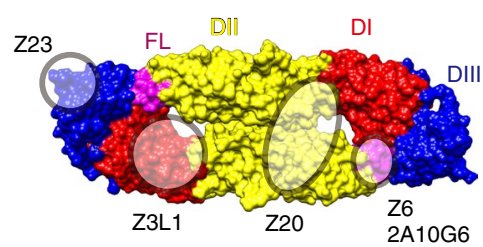

e

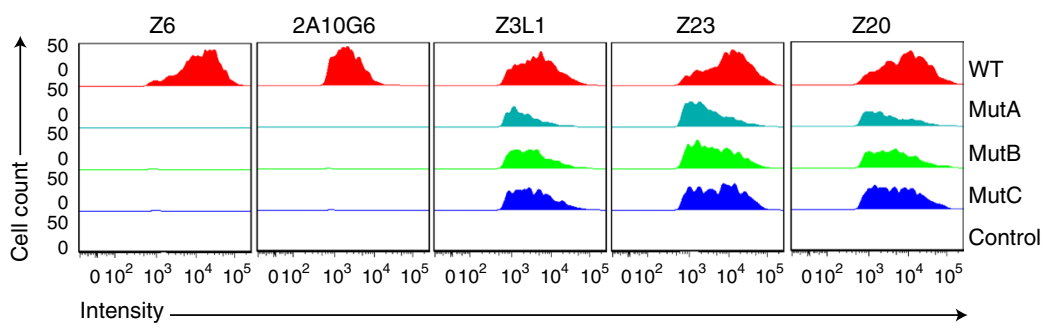

g

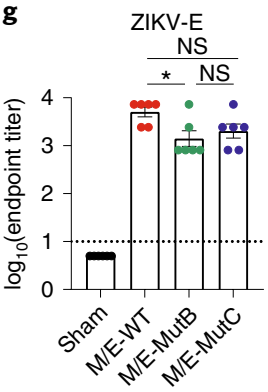

d
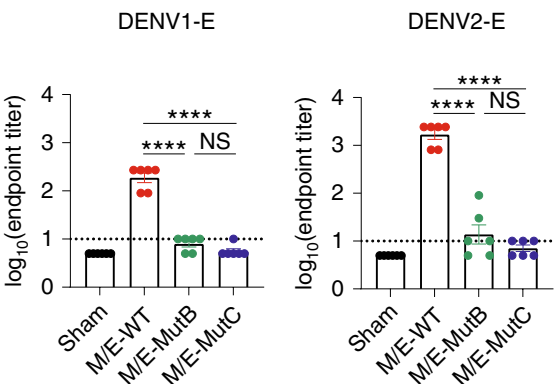

b

\begin{tabular}{|c|c|c|c|}
\hline ZIKV-sE & H Chain & L Chain & $\begin{array}{l}\text { Total } \\
\text { contacts }\end{array}$ \\
\hline P75 & & Y32 (6) ${ }^{a}$ & 6 \\
\hline \multirow{4}{*}{ T76 } & & G28 (2) & \multirow{4}{*}{15} \\
\hline & & $129(3)$ & \\
\hline & & D30 (9) & \\
\hline & & Y32 (1) & \\
\hline \multirow{5}{*}{ W101 } & Y35 (15) & & \multirow{5}{*}{84} \\
\hline & W49 (1) & L91 (5) & \\
\hline & S52 (2) & Y94 (18) & \\
\hline & Y60 (31) & P96 (2) & \\
\hline & Q100 (10) & & \\
\hline G102 & Y35 (1) & & 1 \\
\hline G104 & & Y94 (4) & 4 \\
\hline C105 & & Y94 (10) & 10 \\
\hline \multirow{4}{*}{ G106 } & & L91 (5) & \multirow{4}{*}{34} \\
\hline & & N92 (3) & \\
\hline & & N93 (8) & \\
\hline & & Y94 (18) & \\
\hline \multirow{3}{*}{ L107 } & & Y32 (4) & \multirow{3}{*}{13} \\
\hline & N103 (1) & L91 (5) & \\
\hline & & N92 (3) & \\
\hline \multirow{4}{*}{ F108 } & Y35 (5) & & \multirow{4}{*}{47} \\
\hline & Q100 (2) & Y32 (4) & \\
\hline & G102 (22) & L91 (2) & \\
\hline & N103 (12) & & \\
\hline G109 & & Y32 (5) & 5 \\
\hline K110 & & Y32 (7) & 7 \\
\hline
\end{tabular}

${ }^{a}$ Numbers represent the number of atom-to-atom contacts analyzed by the contact program in CCP4 suite (the distance cutoff is $4.5 \AA$ ).

Fusion loop

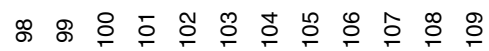

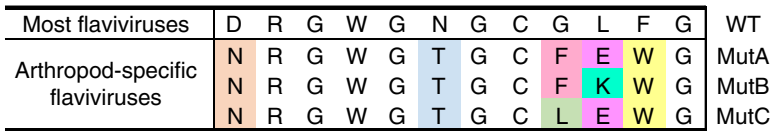
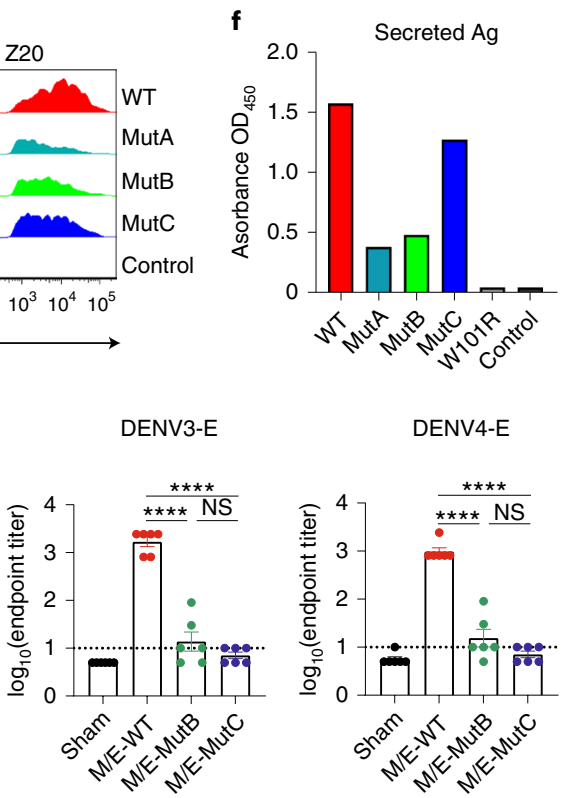
a

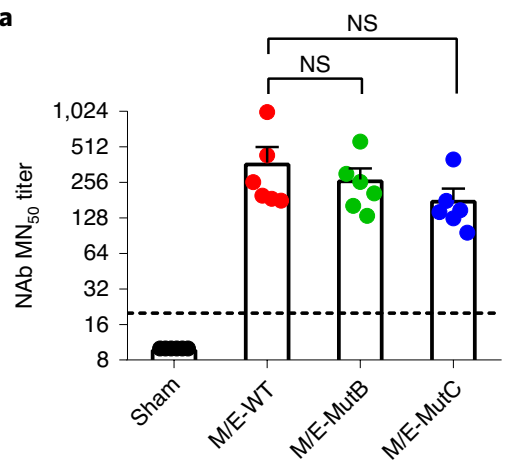

b

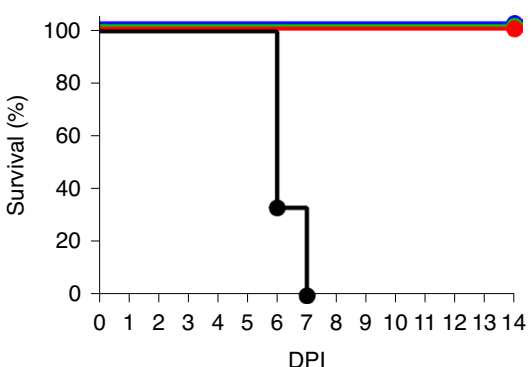

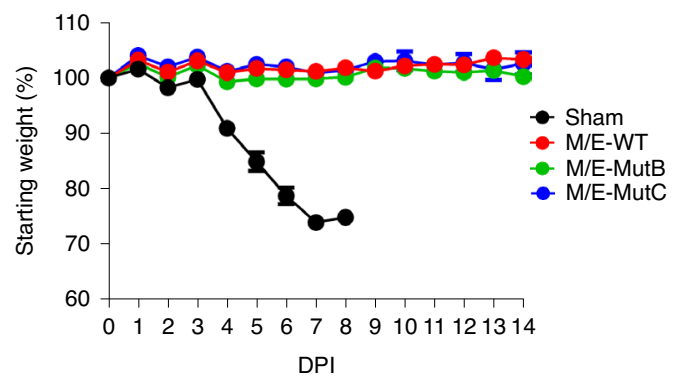

d

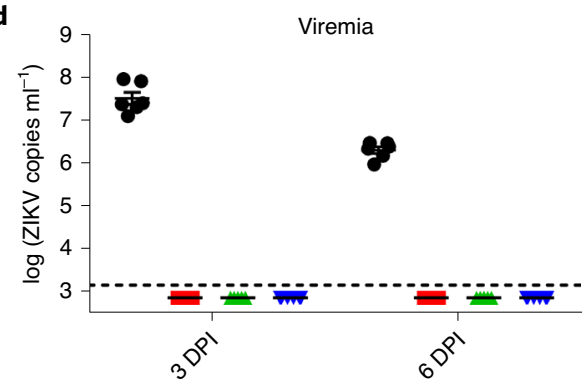

e

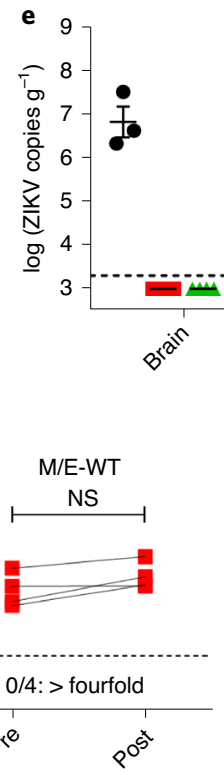

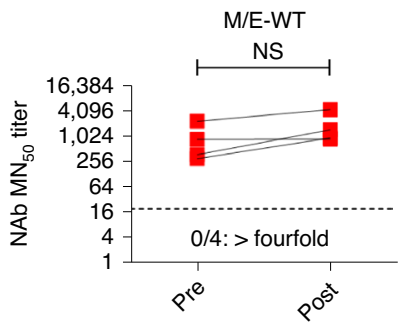

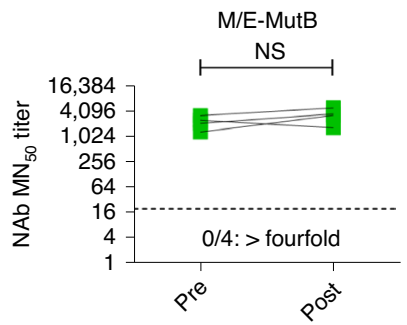

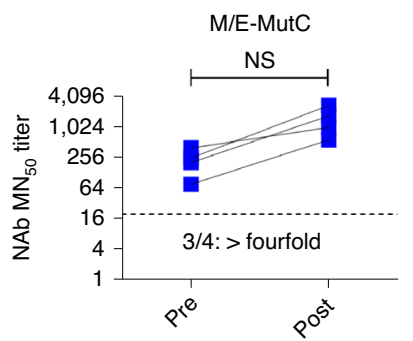

Fig. 2 | Neutralizing activities and protective efficacies of FL-engineered vaccines. a, Sera described in Fig. $1 g$ were measured for neutralizing activities $\left(\mathrm{MN}_{50}\right.$ titers) against ZIKV infection. Data are mean \pm s.e.m. $P$ values were analyzed with two-sided Student's t-test. The dashed line indicates LOD. b-d, Groups of 5-week-old male Ifnar1-/- mice $(n=5-6)$ received one dose $\left(1.6 \times 10^{11} \mathrm{vp}\right)$ of AdC7-M/E-WT, AdC7-M/E-MutB or AdC7-M/E-MutC via the i.m. route. PBS was given as the sham vaccine. At 30 days postimmunization, mice were challenged with $5 \times 10^{6} \mathrm{FFU}$ of $Z \mathrm{IKV}-\mathrm{SMGC}-1$. Animals were monitored daily for survival (b) and weight loss (c). d, Protection by ZIKV vaccines against viremia. Serum samples from mice challenged with ZIKV-SMGC-1 were collected at 3DPI and 6DPI and measured for viral load by qRT-PCR. The dashed line indicates LOD. e,f, Groups of 5-week-old male Ifnar1 ${ }^{-/-}$mice $(n=3-4)$ received a single dose $\left(1.6 \times 10^{11} \mathrm{vp}\right)$ of AdC7-M/E-WT, AdC7-M/E-MutB, AdC7-M/E-MutC or PBS as sham vaccine. At 30 days postvaccination, mice were challenged with $5 \times 10^{6} \mathrm{FFU}$ of ZIKV-SMGC-1 via the i.p. route. e, At 7 DPI, tissues from brain, spinal cord, testis, spleen and liver were harvested for measurement of ZIKV loads. Data are mean \pm s.e.m. The dashed line indicates LOD. f, Anamnestic neutralizing antibody response. Paired serum samples were collected from vaccinated animals 2 days before (Pre) or 7 days after (Post) ZIKV-SMGC- 1 challenge and analyzed for MN $_{50}$ against ZIKV infection. $P$ values were analyzed by paired two-sided Student $t$-test $\left({ }^{\star} P<0.05 ; P\right.$ values are available as source data). Numbers of mice with fourfold NAb titer enhancement are indicated.

of ZIKV-SMGC-1. As expected, all mice receiving the sham vaccine succumbed to ZIKV challenge by 8 days postinfection (DPI), with severe weight loss starting from 4 DPI (Fig. 2b,c). In contrast, all mice receiving the WT- or MutB/C-based vaccines showed no mortality or morbidity (Fig. 2b,c). To validate the protective efficacy of vaccines against viremia, virus burden in blood was measured at both 3 DPI and 6DPI. As expected, mice with sham vaccination displayed sustainable viremia before death. In contrast, all mice receiving WT- or MutB/C-based vaccines showed no detectable viremia (Fig. 2d). To further test protection efficacy of the vaccines against virus burden in ZIKV target tissues, a challenge experiment was performed. A single-dose vaccination elicited binding and neutralizing antibodies (Extended Data Fig. 6a,b). At both days 3 and 7 postchallenge, mice were euthanized for quantification of ZIKV RNA in tissues. Like the WT form, the MutB/C-based vaccines protected mice, with undetectable viral RNA in all tissues tested, including brain, spinal cord, testis, spleen, liver and eye, whereas high viral burdens were detected in sham-vaccinated animals (Fig. 2e and Extended Data Fig. 6c). Given that the FL-engineered vaccines completely protected mice against ZIKV viremia and tissue infection, we further tested whether they conferred sterilizing immunity. Sera collected pre- and post-ZIKV challenge were analyzed for neutralizing activities. Mice immunized with the MutB-based vaccine exhibited a lack of anamnestic responses, as did the WT form, indicating sterilizing immunity (Fig. 2f).

Given that the MutB/C-based vaccines induced antibodies with negligible DENV cross-reactivity (Fig. 1g), we further investigated the ADE potential of these vaccines. Sera from previously vaccinated $\mathrm{BABL} / \mathrm{c}$ mice were further tested for enhancement of virus infection on K562 cells. As expected, the WT-based vaccine induced 

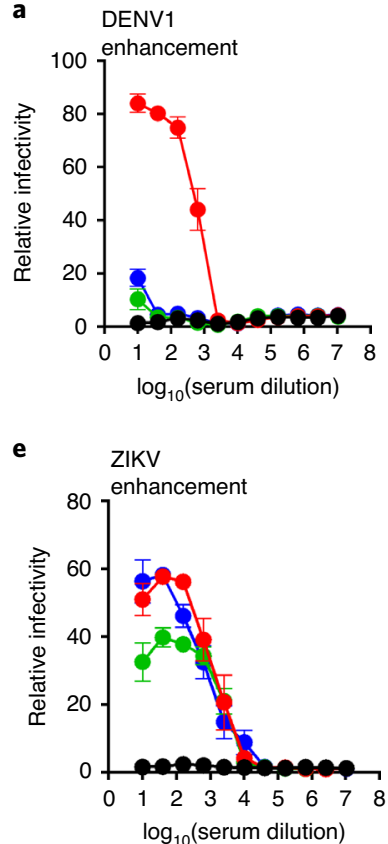
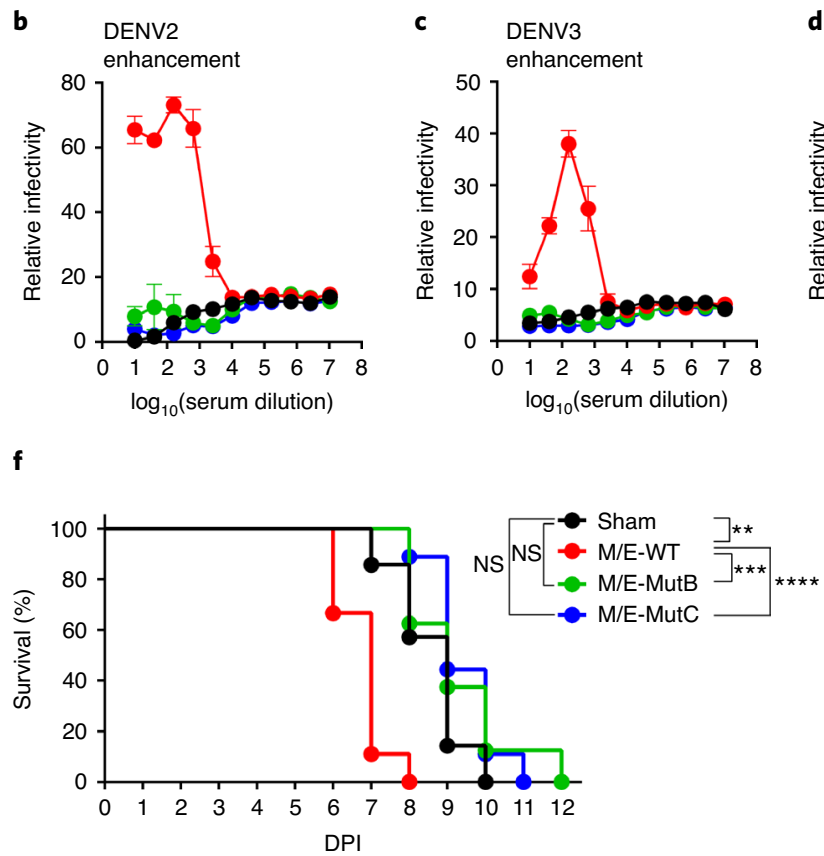
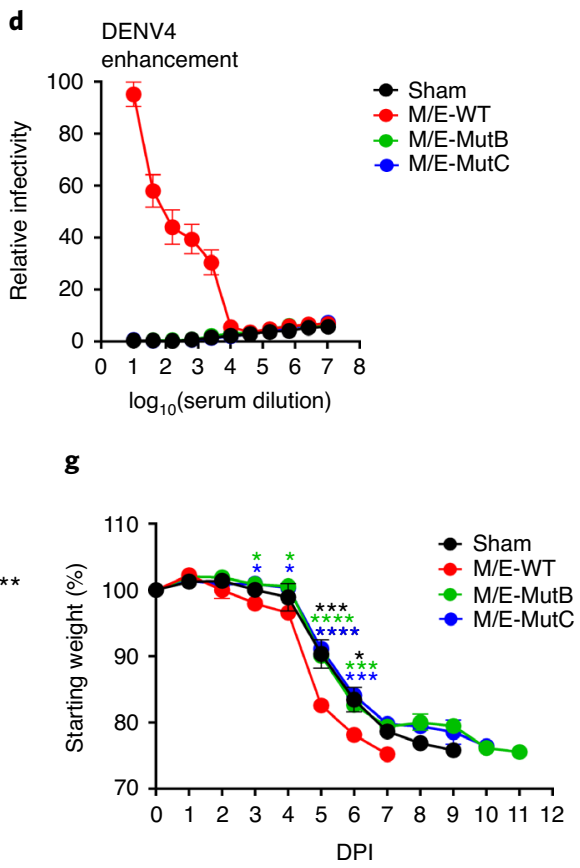

Fig. 3 | Evaluation of ADE effect for FL-engineered vaccines. a-e, ADE activities in cell culture, showing enhancement curves of K562 cells infected with DENV1 (a), DENV2 (b), DENV3 (c), DENV4 (d) and ZIKV (e) in the presence of serially diluted mouse immune sera as indicated. Relative infectivity of each sample was normalized to the peak infectivity of mAb Z5 as shown in Extended Data Fig. 4e. Data are mean \pm s.e.m. $\mathbf{f}, \mathbf{g}$, ADE of DENV infection for pathogenicity in mice. Groups of 6-to-8-week-old female $(n=10)$ BALB/c mice were immunized with a single dose $\left(1.6 \times 10^{11} \mathrm{vp}\right)$ of AdC7-M/E-WT, AdC7-M/E-MutB, AdC7-M/E-MutC or sham as serum donors. Four weeks postimmunization, serum samples were collected and diluted tenfold with PBS before transfer. Next, $200 \mu \mathrm{l}$ of the diluted sera were transferred passively to groups of 5-week-old female and male AG6 mice $(n=7-9)$ via the i.p. route. After $24 \mathrm{~h}, \mathrm{AG} 6$ mice were challenged with 5,000 FFU of DENV2-NGC via the subcutaneous (s.c.) route. Animals were monitored daily for survival (f) and weight loss $(\mathbf{g})$. Results from two independent experiments were pooled. Data are mean \pm s.e.m. for the weight loss. $P$ values for the survival were analyzed with log-rank test; $P$ values for weight loss were analyzed with two-sided Student $t$-test $\left({ }^{\star} P<0.05,{ }^{\star \star} P<0.01,{ }^{\star \star \star} P<0.001,{ }^{\star \star \star \star} P<0.0001 ; P\right.$ values are available as source data).

antibodies capable of enhancing infections of DENV1-4 (Fig. 3ad). However, consistent with the markedly reduced cross-reactivity of serum to DENV, the ADE effect was almost absent in sera from MutB/C groups (Fig. 3a-d). Additionally, we also assessed the infection-enhancing activities of sera for ZIKV. Almost all flavivirus antibodies will induce $\mathrm{ADE}$ against the homologous virus at subneutralizing concentrations ${ }^{11,42}$. Accordingly, we observed either WT- or MutB/C-based vaccines induced serologic responses enhancing ZIKV infection in K562 cells (Fig. 3e). However, sera from MutB-based vaccine showed dramatically decreased ADE of homologous ZIKV infection, with approximately half of the peak enhancement at low dilution (Fig. 3e).

To further interrogate the physiological significance of these results, the vaccine-induced ADE for DENV were tested in vivo by the established passive transfer model in mice deficient in both IFNAR1 and IFNGR (AG6). Donor mice (BALB/c) were immunized with AdC7 expressing M/E-WT, M/E-MutB and M/E-MutC, respectively. $\mathrm{PBS}$ was given as the immune-naïve control. AG6 mice were adoptive transferred with equivalent amounts of pooled sera from groups of donor mice, and were further challenged with 5,000 FFU of DENV2 (NGC strain). The sera of donor mice from MutB/C group produced ZIKV-E-specific IgG and NAbs comparable to those of the WT group (Extended Data Fig. 7a,b), but exhibited no obvious DENV enhancement (Extended Data Fig. 7c). Consistent with the $\mathrm{ADE}$ tendency in vitro, mice receiving sera from donors vaccinated with the WT form succumbed to DENV2-NGC challenge significantly earlier than those receiving sera from the immune-naive or MutB/C-vaccinated donors (WT versus Sham: $P=0.0019$; WT versus MutB: $P=0.0003$; WT versus MutC: $P<0.0001$ ) (Fig. $3 f$ ). In contrast, the death curves between mice receiving donor sera from immune-naïve and MutB/C-vaccinated mice exhibited no significant difference, suggesting no in vivo ADE for DENV2 (Fig. 3f). Consistently, mice receiving sera from donors immunized with the MutB/C-based vaccines did not suffer from severe weight loss compared with those from the immune-naïve group (Fig. $3 \mathrm{~g}$ ).

MutB/C vaccines abrogate maternal-neonatal transmission. Maternal infection with ZIKV can cause a severe congenital syndrome in humans. We therefore sought to evaluate vaccine efficacy for fetal protection against ZIKV challenge in the pregnant mouse model. Groups of female Ifnar $1^{-/}$mice were vaccinated with MutB/C-based, WT-based or sham vaccines (Fig. 4a). Both the MutB/C and WT groups elicited robust ZIKV-E-specific binding and NAb titers (Fig. 4b). These mice were then crossed with male Ifnar1 $1^{-/}$mice overnight for pregnancy. Pregnant mice were then challenged with $1 \times 10^{6} \mathrm{FFU}$ ZIKV-SMGC- 1 via the intraperitoneal (i.p.) route on embryonic day 5.5 (d_e5.5) after observation of a vaginal plug. On d_e13.5, we performed cesarean (C) sections to obtain maternal and fetal tissues from embryos for evaluation (Fig. 4a). As expected, high levels of viral RNA can be detected in maternal brain, spleen and serum from sham-vaccinated mice. In contrast, no viral loads were detectable in these tissues from the MutB/C and WT groups (Fig. 4c). We further measured virus burden in fetal and placental tissues from these vaccinated dams. High titers of virus RNA were detected in both placenta (mean 7.9log copies per gram) and fetal head (mean $5.2 \mathrm{log}$ copies per gram) in 
a
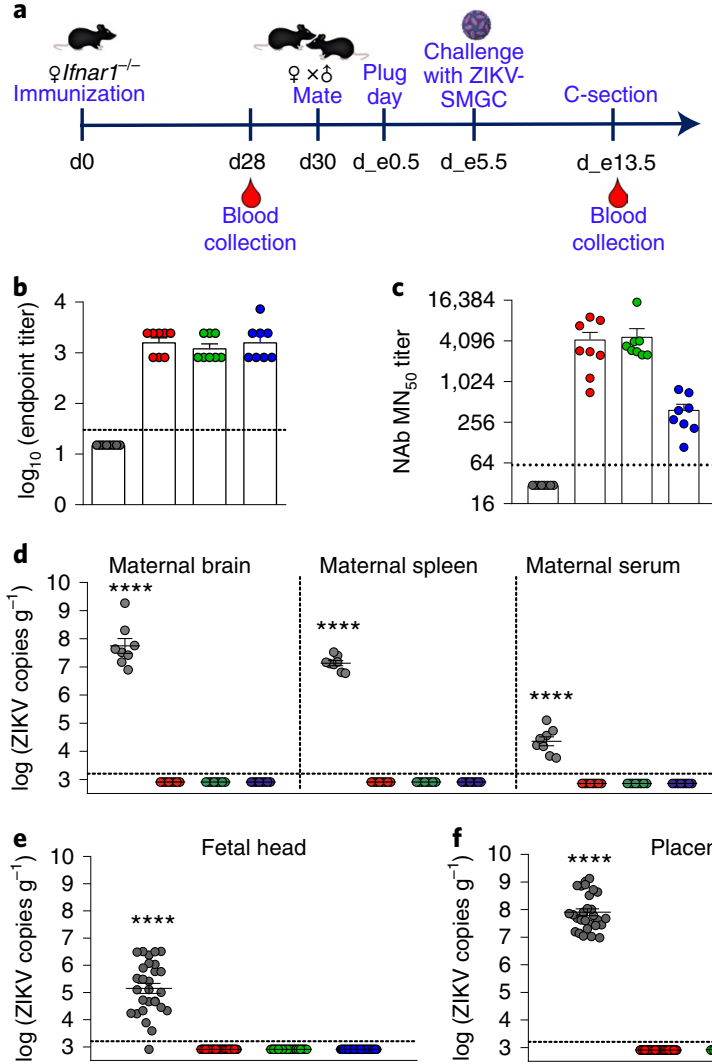

Maternal serum
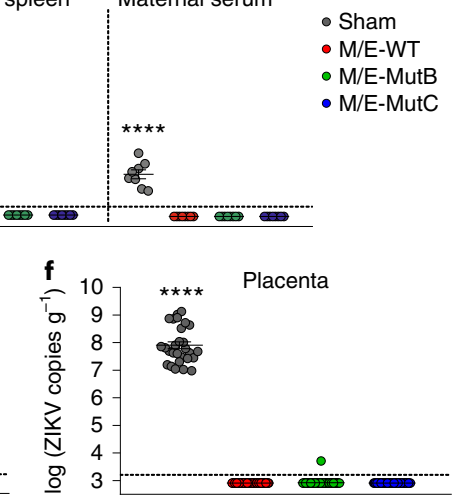

Fig. 4 | MutB/C-based vaccines protect placental and fetal tissues following ZIKV challenge. a, Schematic of study. Groups of 5-to6-week-old female Ifnar ${ }^{-1-}$ mice $(n=8)$ were vaccinated with a single dose $\left(1.6 \times 10^{11} \mathrm{vp}\right)$ of WT-based, MutB/C-based or sham vaccine via the i.m. route. Serum samples were collected on day 28 postvaccination for evaluation of immunogenicity. On day 30 , these dams were crossed with Ifnar $7^{-/-}$males. Pregnant dams were challenged with $5 \times 10^{5} \mathrm{FFU}$ ZIKV-SMGC-1 via the i.p. route at embryotic stage day 5.5 (d_e5.5), followed by harvest of tissues on d_e13.5, including maternal brains, spleens, sera, placentas and fetal heads. b,c ZIKV-E-specific IgG ( $n=8$ biologically independent samples) (b) and NAb titers of maternal sera ( $n=8$ biologically independent samples) (c) collected on day 28. Data are mean \pm s.e.m. The dashed line indicates LOD. $\mathbf{d}-\mathbf{f}$, ZIKV RNA copies were measured for maternal brain, spleen and serum (d), fetal heads (e) and placenta (f). For maternal samples, $n=8$ biologically independent samples. For fetal heads and placenta, $n=28$ biologically independent samples in sham group; $n=54$ biologically independent samples in WT group; $n=52$ biologically independent samples in MutB group, and $n=46$ biologically independent samples in MutC group. $P$ values for virus loads were calculated using two-way ANOVA ( ${ }^{\star \star \star \star} P<0.0001 ; P$ values are available as source data). Data are mean \pm s.e.m.

sham-vaccinated dams. In contrast, no virus load was detected in almost all placentas and heads of pups born to dams vaccinated with MutB/C- or WT-based vaccines (Fig. 4d,e), highlighting the potential of MutB/C-based vaccines to abrogate maternal-neonatal transmission of ZIKV.

MutB/C vaccines switch immunodominance of antibody responses. Given that the MutB/C-based vaccines elicited completely protective immunity without ADE of DENV infection, we further assessed how these vaccines affected antibody responses using $B$ cell profiling at the single-cell level. Three groups of $\mathrm{BALB} / \mathrm{c}$ mice were immunized with WT- or the MutB/C-based vaccines. At 20 days postvaccination, germinal center $\mathrm{B}$ cells $\left(\mathrm{B}_{\mathrm{GC}}\right)$ $\left(\mathrm{GL}_{-} 7^{+} \mathrm{B} 220^{\text {hi }} \mathrm{CD}^{3} 8^{\text {lo }} \mathrm{IgD}^{-} \mathrm{CD}^{-} 3^{-} \mathrm{CD} 138^{-}\right)$from the lymph nodes that bound to ZIKV-E were sorted by flow cytometry (Extended Data Fig. 8). We used a protein mixture of E-monomer/dimer conjugated with fluorochrome to probe the ZIKV-E-reactive $\mathrm{B}_{\mathrm{GC}}$, precluding experiment noise from adenovirus-elicited $\mathrm{B}_{\mathrm{GC}}$. To obtain the paired variable (V)-regions, we applied the chromium immune profiling solution on these ZIKV-E-reactive $\mathrm{B}_{\mathrm{GC}}$ for single-cell B cell receptor sequencing (scBCR-seq) (Fig. 5a). Sequences covering the full-length V(D)J segments for heavy and light chains were selected for further analyses. As a result, from the WT group, 451 variable regions for heavy chain (HV) and 661 variable regions for light chain (LV) were obtained with 334 in pairs; from the MutB group, $310 \mathrm{HV}$ and $379 \mathrm{LV}$ were obtained with 234 in pairs; from the MutC group, $664 \mathrm{HV}$ and $776 \mathrm{LV}$ were obtained with 515 in pairs (Fig. 5b,c and Supplementary Table 2). Impressively, antigen-reactive $\mathrm{B}_{\mathrm{GC}}$ clones elicited by the WT-based vaccine used a narrow pool of $\mathrm{V}$ genes, with $\sim 60 \%$ utilizing IGHV9-2-1, IGHV1-22 or IGHV7-3 for HV, and also 60\% utilizing IGKV10-96, IGKV14-111 or IGKV6-23 for LV (Fig. 5c; Supplementary Table 2). In contrast, antigen-reactive $B_{G C}$ clones elicited by the MutB/C-based vaccines displayed more diverse and dispersed V-gene usages for both HV and LV (Fig. 5c and Supplementary Table 2). The frequency of the dominant $\mathrm{V}$ genes utilized in the WT group were reduced dramatically or absent in the repertoires of MutB/C groups (Fig. $5 \mathrm{c}$ and Supplementary Table 2).

To profile the full $\mathrm{B}$ cell receptor (BCR) usage, we next analyzed the germline genes for the HV and LV in pairs (HV:LV) for these three groups. Consistent with the findings from the respective analyses for $\mathrm{HV}$ and $\mathrm{LV}$, we observed the WT-based vaccine expanded $\mathrm{B}_{\mathrm{GC}}$ clones using the narrow pool of $\mathrm{HV}: \mathrm{LV}$ genes, expressing predominately IGHV9-2-1:IGKV10-96 (29.9\%), IGHV1-22:IGKV14-111 (14.4\%) and IGKV1-22:IGKV6-23 (7.5\%) (Fig. 5d and Supplementary Table 3). In contrast, the MutB/C-based vaccines expanded E-reactive $B_{G C}$ clones using larger pools of $\mathrm{HV}: \mathrm{LV}$ genes with more dispersed gene frequencies (Fig. 5e,f and Supplementary Table 3). In particular, the high-frequent V-gene pairs used in the WT group were absent or minimized in the MutB/C groups. For instance, the most dominant gene pair, IGHV9-2-1:IGKV10-96, was absent in MutB/C groups (Fig. 5e,f and Supplementary Table 3). Taken together, the repertoire profiling suggested that, compared with the WT form, MutB/C-based vaccines switched immunodominance of ZIKV-E-reactive B cell responses.

Main classes of FLE mAbs to enhance DENV infection. To further characterize the antibodies elicited from these three vaccination groups, a panel of antibody genes was synthesized, representing the top most frequent clusters of genetically similar $\mathrm{B}_{\mathrm{GC}}$ clones, for $63.4 \%$ of the WT group, $46.6 \%$ of the MutB group and $43.9 \%$ of the MutC group (Supplementary Table 4 ). The HV and LV sequences were then cloned into mouse IgG2a and Igא expression vectors, respectively. The mAbs derived from the WT, MutB and MutC groups were termed ZWT.1-10, ZMutB.1-8 and ZMutC.1-13, respectively (Supplementary Table 4). We first tested their binding properties to ZIKV-E by ELISA. Most mAbs can bind to soluble $\mathrm{E}(\mathrm{sE})$-monomer/dimer, with a frequency of $90 \%$ (9/10) in the WT group, $87.5 \%$ (7/8) in the MutB group and $76.9 \%$ $(10 / 13)$ in the MutC group (Fig. 5b and Supplementary Table 4), suggesting the reliability of our approach. The $26 \mathrm{E}$-binding mAbs were further evaluated. Notably, we found that none of the mAbs derived from the WT group interacted with ZIKV-sE-MutC, but were cross-reactive with serotypes of DENV-sE, implying characteristics of FLE mAb (Fig. 6a). However, mAbs derived from the MutB/C groups had almost no-binding to DENV1-4 (Fig. 6a). 
Next, representative $\mathrm{mAbs}$ in each group were tested for their ADE effects for DENV infections on K562 cells. Consistently, the dominant $\mathrm{mAbs}$ derived from WT-vaccinated mice largely enhanced DENV1-4 infections, whereas the unrelated $\mathrm{mAb}$ did not (Fig. $6 \mathrm{~b}-\mathrm{e})$. In contrast, the dominant $\mathrm{mAbs}$ from $\mathrm{MutB} / \mathrm{C}$-vaccinated mice did not show ADE for any of the four serotypes of DENV (Fig. 6f-i).

The FLE mAbs elicited from the WT group used predominantly four classes of HV:LV gene pairs (Fig. 6j). Next, we sought to analyze the publicly available sequence for murine FLE $m A b s$ and found four such mAbs: 6B6C-1 from St. Louis encephalitis virus (SLEV)-immune ${ }^{43}, 4 \mathrm{G} 2$ and 2A10G6 from DENV-immune ${ }^{44,45}$ and E53 from WNV-immune ${ }^{46}$. Interestingly, these four $\mathrm{mAbs}$ were genetically close to the four main classes of FLE mAbs in the WT group (Fig. 6j). 6B6C-1 and 4G2 used the same HV:LV genes as ZWT.1-3 and ZWT4-5, respectively (Fig. 6j), with high sequence similarities (Extended Data Figs. 9a-d). 2A10G6 and E53 used the same HV gene as ZWT.6 and ZWT.8, respectively (Fig. 6j). Besides, 2A10G6 shared exactly the same amino acids with ZWT.6 in CDRL3, a region found to determine the FL-binding for light chain of 2A10G6 (Extended Data Fig. 9e) ${ }^{35}$. Taken together, flavivirus FLE mAbs, isolated from mice with ZIKV-, DENV-, SLEV- and WNV-immune, were found to use almost the same few germline genes.

Structural basis of the FL-engineered immunogen. Since the FL-engineered vaccines were completely protective, with elimination of ADE for DENV in mice, we sought to explore the underlying molecular basis. ZIKV-sE-MutC were expressed and purified as representative for evaluation. A surface plasmon resonance (SPR) experiment was first performed to evaluate the binding affinities of sE-MutC to FLE and non-FLE mAbs with sE-WT tested as a control. Consistently, either the DI or DIII targeting $\mathrm{mAb}$ (Z3L1 or Z23) bound to sE-MutC with affinity (equilibrium dissociation constant $\left(K_{\mathrm{D}}\right)$ ) almost equivalent to its binding to sE-WT, indicating the stable presentation of neutralizing epitopes. In contrast, binding between sE-MutC and FLE mAbs (Z6 and 2A10G6) was undetectable (Fig. 7a and Supplementary Fig. 1), suggesting FLE disruption.

To further explore the molecular basis of vaccine potency and ADE abrogation at the atomic level, we determined the crystal structure of sE-MutC bound to Z3L1 at $3.1 \AA$ resolution (Supplementary Table 1). Although five-residue mutations were introduced into FL, sE-MutC was still arranged as an E-dimer to engage Z3L1 in its prefusion state (Extended Data Fig. 10a). When folded, it resembled its WT form (root mean squared (r.m.s.) deviation of $1.04 \AA$ for their $\mathrm{C}_{\alpha}$ positions), showing the normal secondary, tertiary and quaternary structures, indicating the stable display of neutralizing epitopes (Fig. 7b). The sE-MutC dimer presented neutralizing epitopes as E proteins in mature ZIKV virions (Extended Data Fig. 10b). Interface analysis revealed the interaction network used to maintain dimer formation (Supplementary Table 5). In particular, compared with the WT form, FL-MutC built alternative interactions with the adjacent $\mathrm{E}$ protomer, introducing four potential hydrogen bonds with the neighboring DI/DIII residues (three by
D98N and one by F108W) (Fig. 7c,d and Supplementary Table 5). Superimposing the structure of FL-MutC on its WT form, we found both FLs were arranged as similar conformations (r.m.s. deviation of $0.35 \AA$ for their $\mathrm{C}_{\alpha}$ positions), with only side-chain variations at mutated sites (Fig. $7 \mathrm{~b}$ ). To further interpret the potential elimination of FLE-antibody induction, we superimposed the DII structure of sE-MutC with its homologs from the structure-known complexes of FLE mAb bound to flavivirus-E. Notably, while the FL-WT was well accommodated by cavities of these FLE mAbs, mutations at G106, L107 and F108 would obviously interfere with reciprocal docking (Fig. 7e-g). The long side-chain protruding from G106L causes severe clashes for all these FLE mAbs (Fig. 7e-g). Moreover, L107E generated a charged side-chain and may destroy the local hydrophobic interactions that existed in FL-WT bound to these FLE mAbs (Fig. 7e-g). Besides, F108W also causes steric hindrance for 2A10G6 (Fig. 7f). Taken together, these three mutations contribute synergistically to abrogation of the elicitation of FLE mAbs.

\section{Discussion}

Given increasing knowledge of the long-term negative impact on children born with confirmed ZIKV infection during pregnancy in humans ${ }^{47}$, sterilizing immunity may be required to completely prevent viral seeding of the placenta and fetal infection ${ }^{13,33}$. Previous studies have described that the introduction of FLE mutations (including W101R) into ZIKV vaccine dramatically reduced $\mathrm{ADE}$ for DENV. However, these mutations sacrificed neutralizing titers significantly compared with the unmutated construct and, as a result, protection against viremia and tissue infections was incomplete ${ }^{13}$. Mutations at W101 impaired the antigen's ability to stably present neutralizing epitopes on $\mathrm{E}$ domains (Extended Data Fig. 3), and are also likely to disrupt E-dimer formation, with the E-dimer epitope (EDE) destroyed ${ }^{11}$. We designed mutations based on naturally occurring FL variants within members of the genus Flavivirus that maintained structural stability. These mutations retained the neutralizing epitopes presented on E domains, and also maintained E-dimer formation to present the potential EDE. Indeed, the MutB/C-based vaccines elicited E-dimer-dependent or -preferred antibodies, represented as mAbs ZMutB.2, ZMutB.3, ZMutB.4, ZMutB.7 and ZMutC.8 (Fig. 6a). Given that EDEs are targeted by potent DENV/ZIKV $\mathrm{NAbs}^{11,26,28,48}$, keeping the E-dimer conformation may be important for vaccine potency. The strategy to retain EDE is also a goal of DENV vaccine with ADE abrogation ${ }^{49}$.

Whether the antigen is soluble or particulate needs to be further determined. However, we propose that intracellular and secreted antigens can stimulate B/T cell responses. Also, intracellular antigens are certainly targets processed by APC for T cell activation.

The L107K in MutB are proposed to exert a similar repulsive effect to interfering with reciprocal binding by FLE mAbs. Therefore, we propose that MutB and MutC may have similar mechanisms to destroy FLE. The structural analysis provides a basis for the rational design of DENV vaccine devoid of ADE. A previous study with a DENV1 DNA plasmid vaccine reduced the ADE

Fig. 5 | Profiling of B cell repertoire by scBCR-seq to dissect the immune responses induced by WT- or MutB/C-based vaccines. Groups of 6-to8-week-old female BALB/c mice $(n=10)$ were immunized with AdC7-M/E-WT, AdC7-M/E-MutB or AdC7-M/E-MutC. Lymph nodes from mice were collected, and single-cell suspensions were prepared. ZIKV-E (mixture of both monomer and dimer)-positive $B_{\mathrm{GC}}$ were sorted by flow cytometry. a, Schematic representation of chromium single-cell immune profiling solution to obtain paired and full-length $V(D) J$ sequences of immunoglobulin (Ig). b, Summary of statistics for scBCR-seq. c, Pie charts show clonal expansion of ZIKV-E-reactive B $B_{G C}$ cells for IGHV (upper panel) or IGLV (bottom panel). Colored slices are proportional to the number of clonal relatives. White indicates the $V$ gene sequences with frequencies less than 3 . See also related Supplementary Table 2. d-f, Paired V-gene use in B cell repertoires induced by ZIKV vaccines based on WT (d), MutB (e) or MutC (f). Surface maps shows the paired HV:LV. V genes are plotted in alphanumeric order, with heights indicating percentage representation among each group. The surface maps were produced by OriginPro. See also related Supplementary Table 3. 
of DENV2 infection ${ }^{50}$. Analogously, in that study, two mutations (a long side-chain G106R and a charged side-chain L107D) were introduced.
Here, we provide effective and safe Zika vaccine candidates to prevent both ZIKV infection and infection/vaccination-induced severe dengue disease. We used a $1.6 \times 10^{11}$ virus particles (vp) dose vaccine

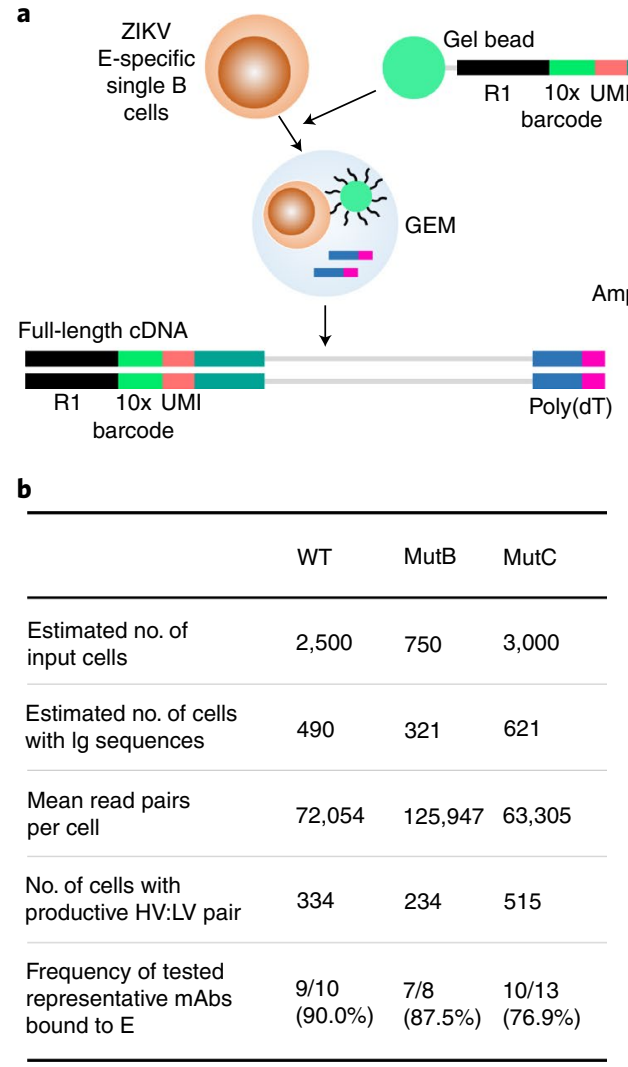

d

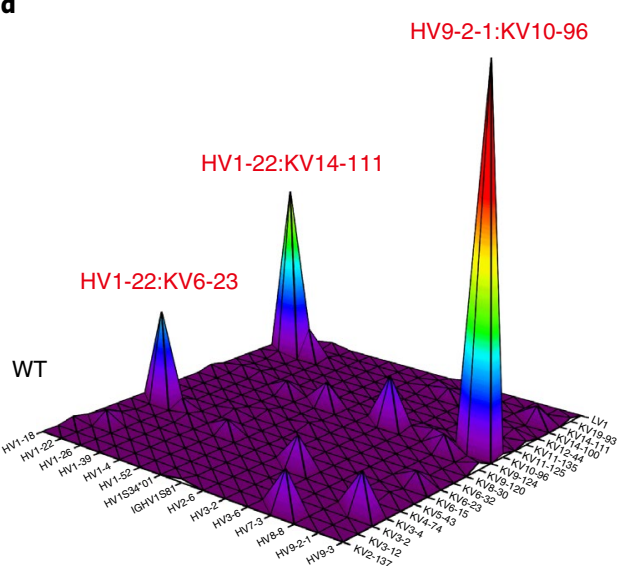

Amplification

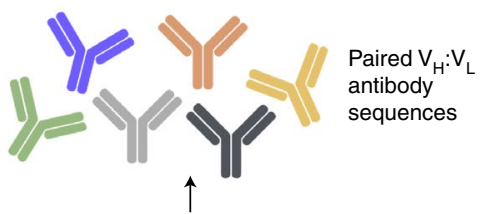

Sequencing and analysis

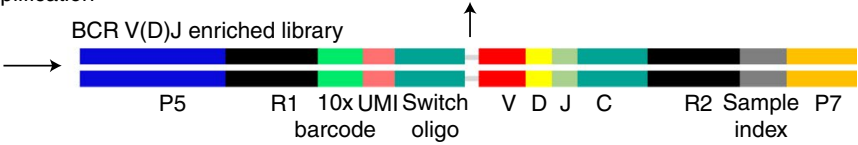

C
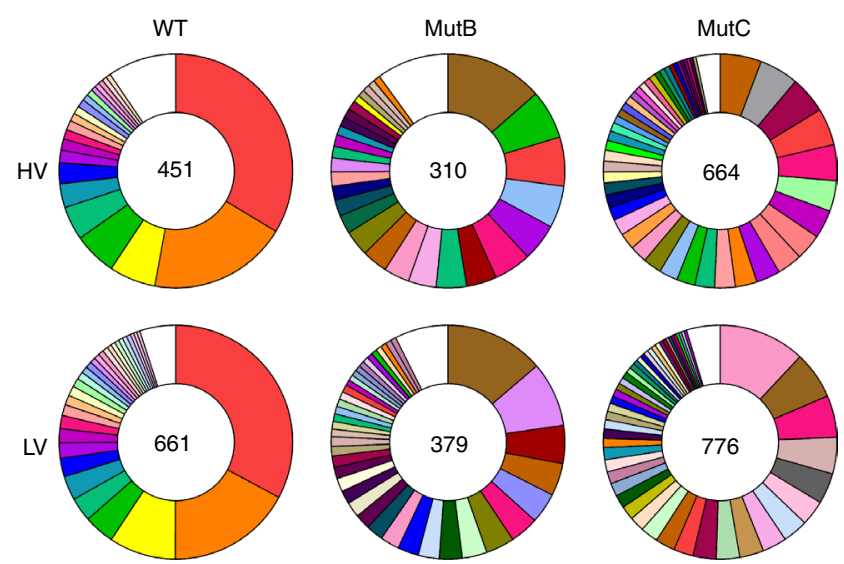

e

Repertoire (\%)

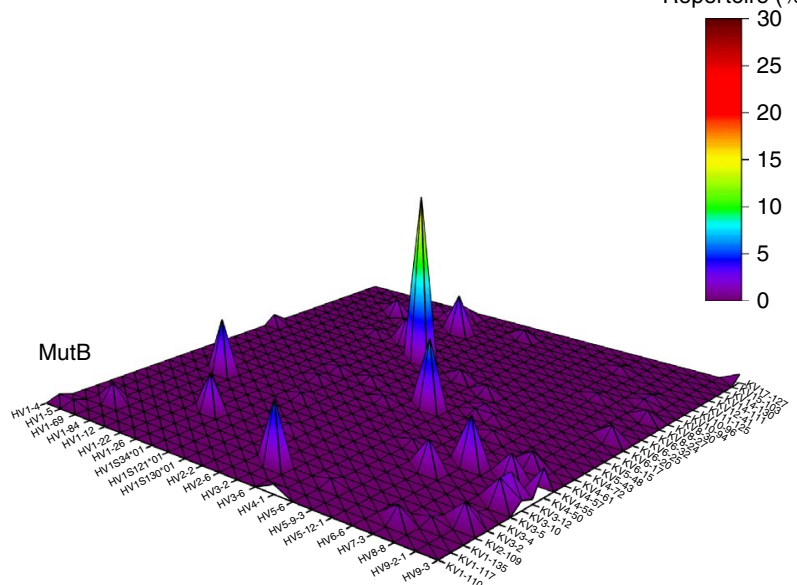

f

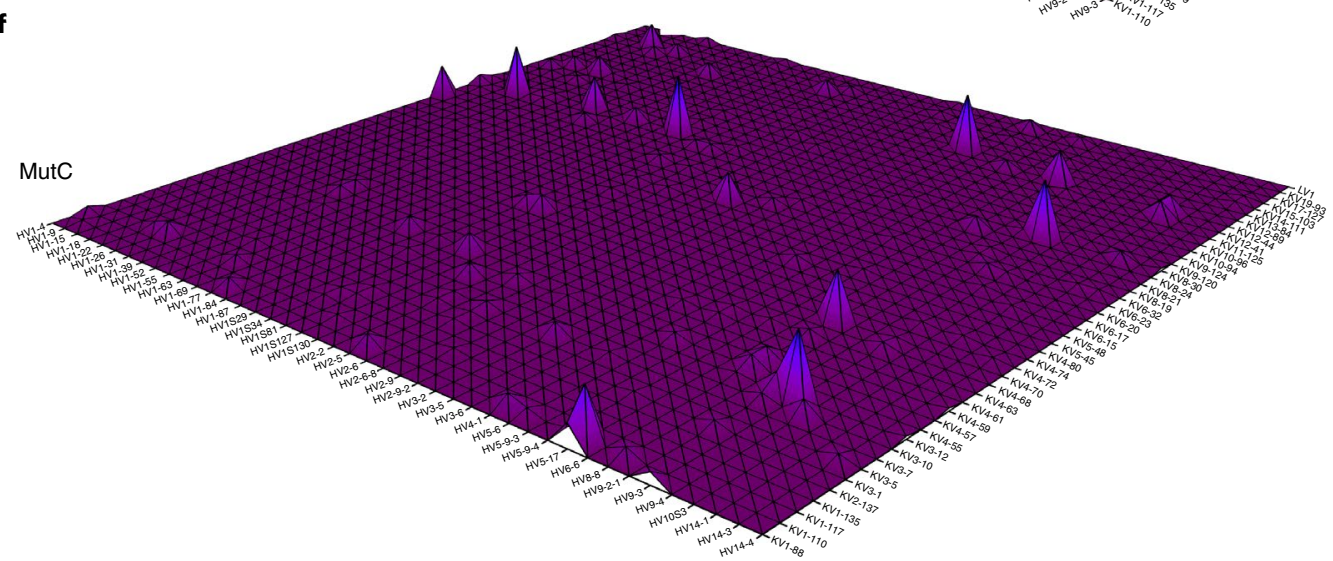


a

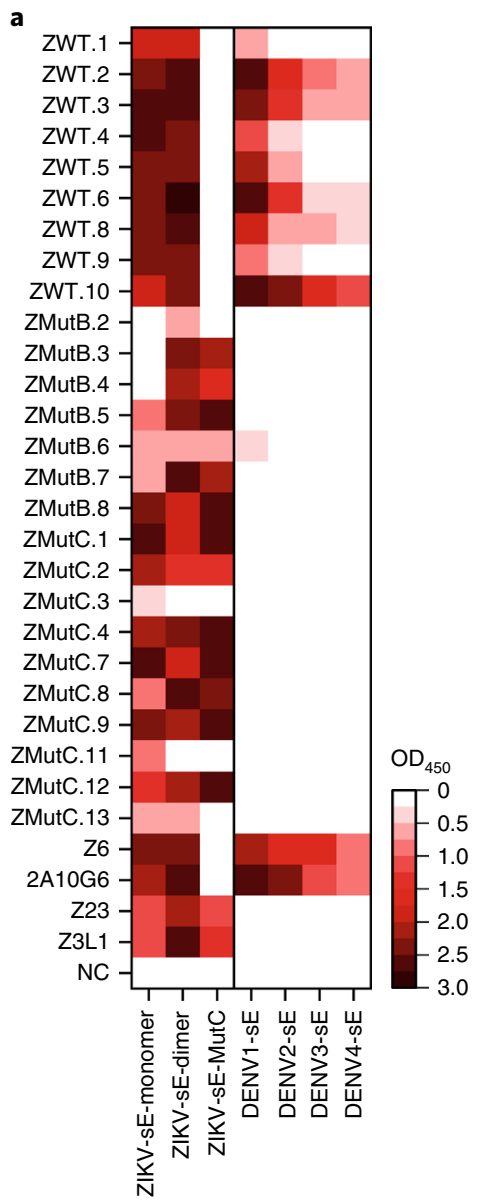

j

\begin{tabular}{|c|c|c|}
\hline FLE mAbs & HV gene & LV gene \\
\hline ZWT.1 & \multirow{4}{*}{ IGHV9-2-1*01 } & \multirow{4}{*}{ IGKV10-96*01 } \\
\hline ZWT.2 & & \\
\hline ZWT.3 & & \\
\hline $6 \mathrm{~B} 6 \mathrm{C}-1$ & & \\
\hline ZWT.4 & \multirow{3}{*}{ IGHV1-22*01 } & \multirow{3}{*}{ IGKV14-111*01 } \\
\hline ZWT.5 & & \\
\hline $4 \mathrm{G} 2$ & & \\
\hline ZWT.6 & \multirow{2}{*}{ IGHV1-22*01 } & IGKV6-23*01 \\
\hline $2 \mathrm{~A} 10 \mathrm{G} 6$ & & IGKV6-13*01 \\
\hline ZWT.8 & \multirow{2}{*}{ IGHV7-3*02 } & IGKV3-12*01 \\
\hline E53 & & IGKV4-59*01 \\
\hline
\end{tabular}

b

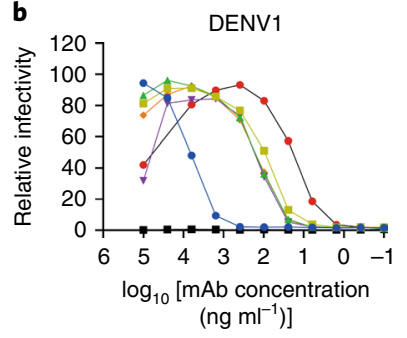

d

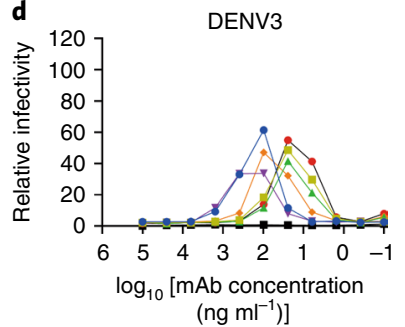

f
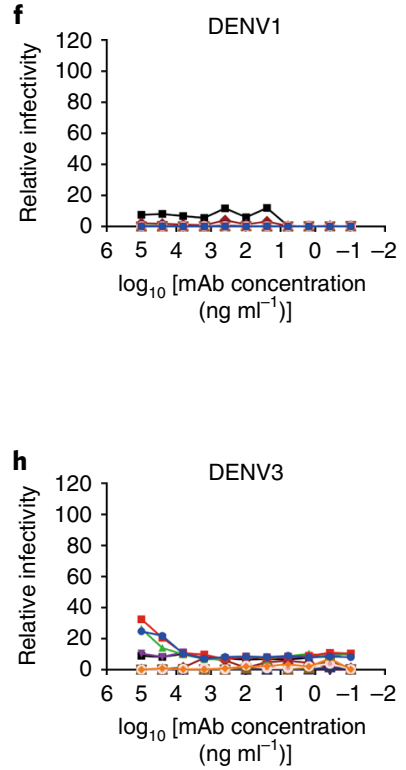

c

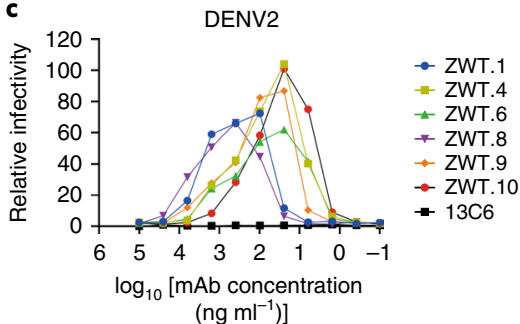

e

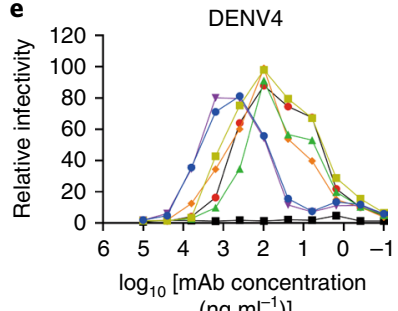

$\left(\mathrm{ng} \mathrm{ml}^{-1}\right)$ ]
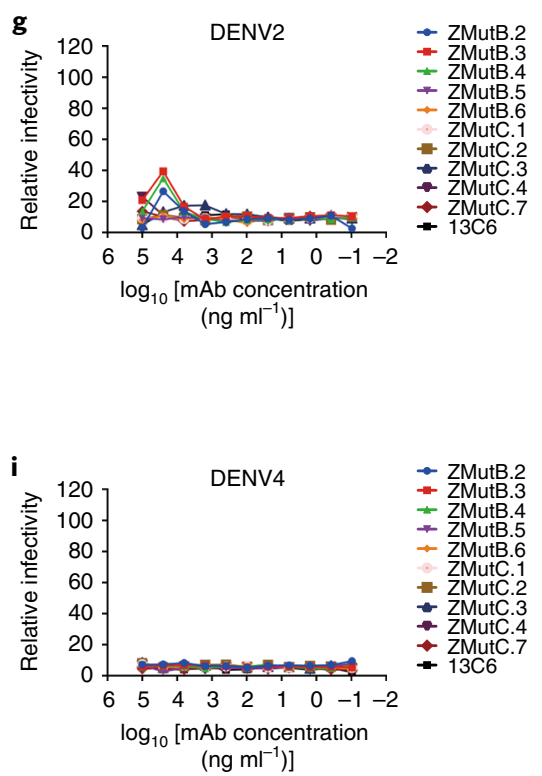

Fig. 6 | Characterization of representative mAbs elicited in mice at high frequency by WT- or MutB/C-based ZIKV vaccines. a, Heatmap illustrating ELISA binding $\left(\mathrm{OD}_{450}\right)$ of 26 ZIKV-E-binding mAbs to ZIKV-sE monomer, dimer, sE-MutC and sE from DENV1-4. The mAbs are derived from the supernatants of HEK293 cells cotransfected with plasmids expressing Ig heavy and light chains for each mAb. b-i, ADE activities of the dominant mAbs derived from mice immunized with WT-based (b-e) or MutB/C-based (f-i) vaccine. Shown are enhancement curves of K562 cells infected with DENV1 (b,f), DENV2 (c,g), DENV3 (d,h) and DENV4 (e,i) in the presence of serially diluted mAbs as indicated. Ebola-virus-specific mAb $13 \mathrm{C} 6$ was used as the negative control. Relative infectivity for each sample was normalized to the peak infectivity of mAb Z6. j, Germline analyses for the FLE mAbs. mAbs ZWT.1-3 use the same HV:LV as 6B6C-1; mAbs ZWT.4-5 use the same HV:LV as 4G2. mAb ZWT.6 uses the same HV gene as 2A10G6, and ZWT.8 uses the same HV gene as E53. The mAbs with known structure bound to flavivirus-E are highlighted in green. The HV and LV genes predominating in the WT-vaccinated group are highlighted with colors as described in Fig. $5 \mathrm{c} .{ }^{\star} 01$ and ${ }^{\star} 02$ indicate the IGHV/ IGKV allele.

in this proof-of-concept study; however, the minimum dose should be explored as our next step in vaccine development. MutB/C could be applied to other vaccine platforms, such as DNA, messenger RNA, different virus vectors or subunit proteins. This study in a mouse model provided immunological trends for vaccine evaluation in humans and highlighted attractive candidates for further development. The global analysis of analogous amino acid sequences from members of a whole genus affords new insight into vaccine-oriented protein design.

\section{Online content}

Any methods, additional references, Nature Research reporting summaries, source data, extended data, supplementary information, acknowledgements, peer review information; details of 


\begin{tabular}{lll} 
a & \multicolumn{2}{c}{ Apparent binding affinity $\left[K_{\mathrm{D}}(\mathrm{M})\right]$} \\
\hline & sE-WT & sE-MutC \\
\hline Z3L1 & $9.48 \times 10^{-6}$ & $8.01 \times 10^{-6}$ \\
\hline Z23 & $6.25 \times 10^{-7}$ & $7.01 \times 10^{-7}$ \\
\hline Z6 & $7.14 \times 10^{-9}$ & - \\
\hline 2A10G6 & $9.13 \times 10^{-9}$ & - \\
\hline
\end{tabular}

b

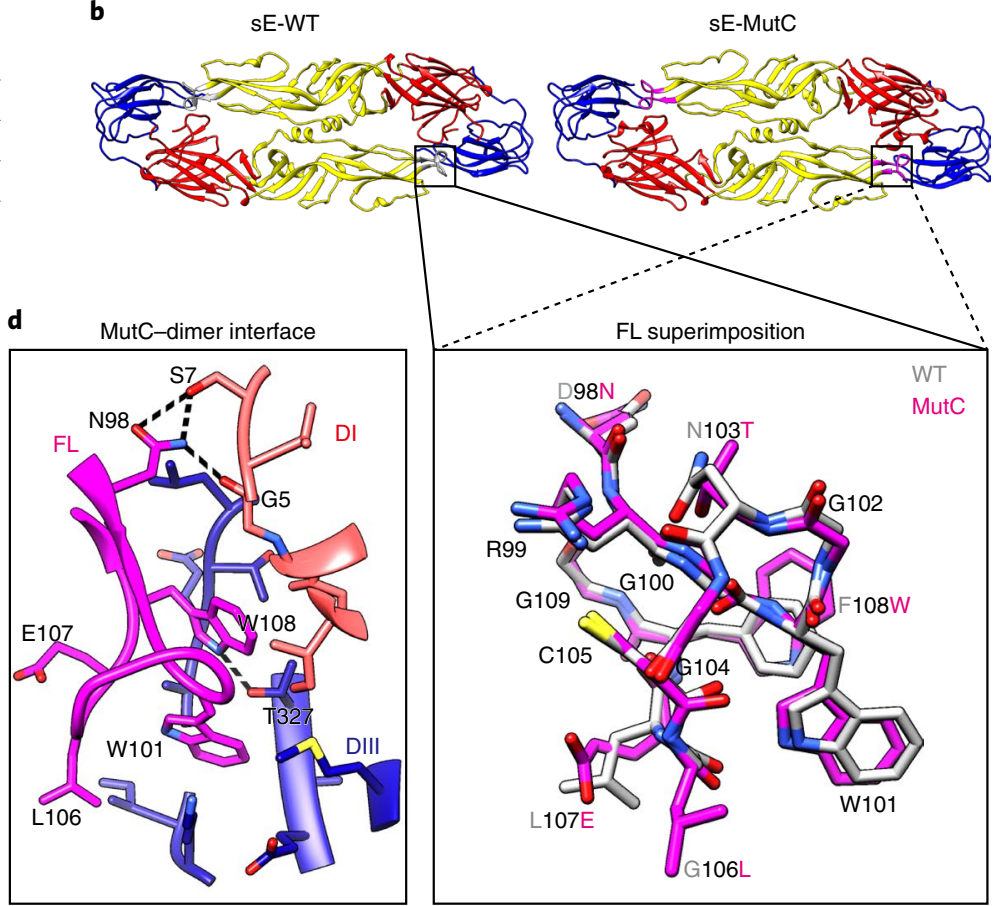

c
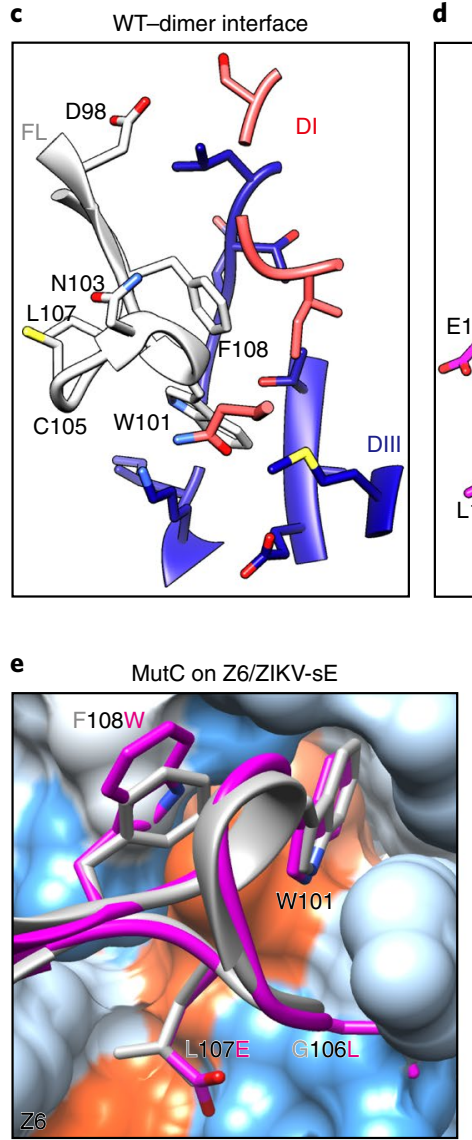

d f

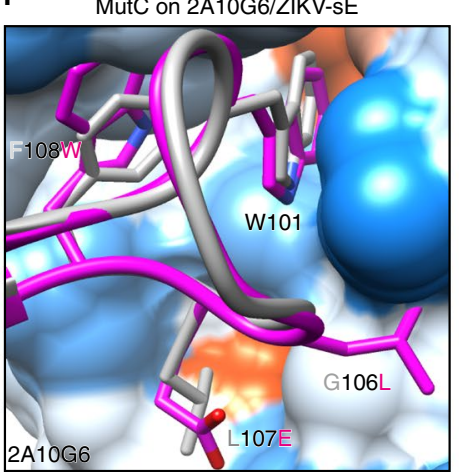

g

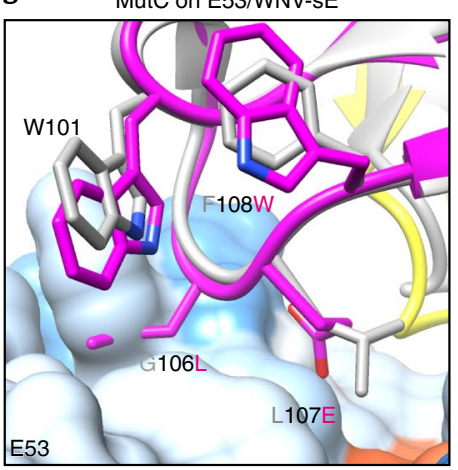

Fig. 7 | Structural characterization of the FL-engineered immunogen. a, Binding affinities of Fab of Z3L1, Z23, Z6 or 2A10G6 bound to sE-WT or sE-MutC. Fab of Z6 and 2A10G6 have no detectable binding activities with sE-MutC. See also the BIAcore diagram in Supplementary Fig. 1. b, Crystal structure of sE-MutC and sE-WT (PDB 5GZN) shown as ribbon. Both FL structures are superimposed with their residues shown as atom/bonds. c,d, Dimer interface of ZIKV-E between FL and its reciprocal E protomer. The interactive residues are shown as ribbons and atoms/bonds. Residues in FL are labeled. The WT-dimer interface is shown in c and MutC-dimer interface is shown in $\mathbf{d}$. Dashed lines represent hydrogen bonds, with the residues labeled. e-g, FL of sE-MutC is superimposed onto the complex structures of FLE mAb/E on Z6/ZIKV-sE (e), on 2A10G6/ZIKV-sE (PDB 5JHL) (f) and on E53/WNV-sE (PDB 3150) (g). The antibodies are shown as hydrophobicity surfaces, displaying amino acid hydrophobicity on the Kyte-Doolittle scale, with colors ranging from bright-blue for the most hydrophilic, to white at 0.0, and to orange-red for the most hydrophobic. Key residues in FL are shown as atoms/bonds and labeled.

author contributions and competing interests; and statements of data and code availability are available at https://doi.org/10.1038/ s41590-021-00966-6.

Received: 24 February 2021; Accepted: 26 May 2021;

Published online: 15 July 2021

\section{References}

1. Knipe, D. M., Howley, P. M. (eds) Fields Virology 6th edn (Lippincott Williams \& Wilkins Health, 2013)

2. Baud, D., Gubler, D. J., Schaub, B., Lanteri, M. C. \& Musso, D. An update on Zika virus infection. Lancet 390, 2099-2109 (2017).

3. Wang, Q. et al. Genetic and biological characterization of Zika virus from human cases imported through Shenzhen Port. Chin. Sci. Bull. 61, 2463-2474 (2016)
4. Brasil, P. et al. Zika virus infection in pregnant women in Rio de Janeiro. N. Engl. J. Med. 375, 2321-2334 (2016).

5. Rasmussen, S. A., Jamieson, D. J., Honein, M. A. \& Petersen, L. R. Zika virus and birth defects-reviewing the evidence for causality. N. Engl. J. Med. 374, 1981-1987 (2016)

6. van der Eijk, A. A. et al. Miscarriage associated with Zika virus infection. N. Engl. J. Med. 375, 1002-1004 (2016).

7. Cao-Lormeau, V. M. et al. Guillain-Barre Syndrome outbreak associated with Zika virus infection in French Polynesia: a case-control study. Lancet 387, 1531-1539 (2016).

8. Miner, J. J. \& Diamond, M. S. Zika virus pathogenesis and tissue tropism. Cell Host Microbe 21, 134-142 (2017).

9. Ma, W. et al. Zika virus causes testis damage and leads to male infertility in mice. Cell 167, 1511-1524.e10 (2016).

10. Govero, J. et al. Zika virus infection damages the testes in mice. Nature $\mathbf{5 4 0}$, 438-442 (2016). 
11. Slon-Campos, J. L. et al. A protective Zika virus E-dimer-based subunit vaccine engineered to abrogate antibody-dependent enhancement of dengue infection. Nat. Immunol. 20, 1291-1298 (2019).

12. Fowler, A. M. et al. Maternally acquired Zika antibodies enhance dengue disease severity in mice. Cell Host Microbe 24, 743-750.e5 (2018).

13. Richner, J. M. et al. Modified mRNA vaccines protect against Zika virus infection. Cell 168, 1114-1125.e10 (2017).

14. Rey, F. A., Stiasny, K., Vaney, M. C., Dellarole, M. \& Heinz, F. X. The bright and the dark side of human antibody responses to flaviviruses: lessons for vaccine design. EMBO Rep. 19, 206-224 (2018).

15. Halstead, S. B. \& O'Rourke, E. J. Dengue viruses and mononuclear phagocytes. I. Infection enhancement by non-neutralizing antibody. J. Exp. Med. 146, 201-217 (1977).

16. Katzelnick, L. C. et al. Antibody-dependent enhancement of severe dengue disease in humans. Science 358, 929-932 (2017).

17. Halstead, S. B. Dengvaxia sensitizes seronegatives to vaccine enhanced disease regardless of age. Vaccine 35, 6355-6358 (2017).

18. Sridhar, S. et al. Effect of dengue serostatus on dengue vaccine safety and efficacy. N. Engl. J. Med. 379, 327-340 (2018).

19. Stettler, K. et al. Specificity, cross-reactivity, and function of antibodies elicited by Zika virus infection. Science 353, 823-826 (2016).

20. George, J. et al. Prior exposure to Zika virus significantly enhances peak Dengue-2 viremia in rhesus macaques. Sci. Rep. 7, 10498 (2017).

21. Katzelnick, L. C. et al. Zika virus infection enhances future risk of severe dengue disease. Science 369, 1123-1128 (2020).

22. York, A. Zika virus enhances dengue risk. Nat. Rev. Microbiol. 18, 605 (2020).

23. Rogers, T. F. et al. Zika virus activates de novo and cross-reactive memory B cell responses in dengue-experienced donors. Sci. Immunol. 2, eaan6809 (2017).

24. Dejnirattisai, W. et al. Cross-reacting antibodies enhance dengue virus infection in humans. Science 328, 745-748 (2010).

25. Beltramello, M. et al. The human immune response to Dengue virus is dominated by highly cross-reactive antibodies endowed with neutralizing and enhancing activity. Cell Host Microbe 8, 271-283 (2010).

26. Dejnirattisai, W. et al. A new class of highly potent, broadly neutralizing antibodies isolated from viremic patients infected with dengue virus. Nat. Immunol. 16, 170-177 (2015).

27. Dai, L., Wang, Q., Song, H. \& Gao, G. F. Zika virus envelope protein and antibody complexes. Subcell. Biochem. 88, 147-168 (2018).

28. Barba-Spaeth, G. et al. Structural basis of potent Zika-dengue virus antibody cross-neutralization. Nature 536, 48-53 (2016)

29. Rouvinski, A. et al. Recognition determinants of broadly neutralizing human antibodies against dengue viruses. Nature 520, 109-113 (2015).

30. Fibriansah, G. \& Lok, S. M. The development of therapeutic antibodies against dengue virus. Antivir. Res. 128, 7-19 (2016).

31. Wang, Q. et al. Molecular determinants of human neutralizing antibodies isolated from a patient infected with Zika virus. Sci. Transl. Med. 8, 369ra179 (2016).

32. Zhao, H. et al. Structural basis of Zika virus-specific antibody protection. Cell 166, 1016-1027 (2016).

33. Abbink, P., Stephenson, K. E. \& Barouch, D. H. Zika virus vaccines. Nat. Rev. Microbiol. 16, 594-600 (2018).
34. Xu, K. et al. Recombinant chimpanzee adenovirus vaccine AdC7-M/E protects against Zika virus infection and testis damage. J. Virol. 92, e01722-17 (2018).

35. Dai, L. et al. Structures of the Zika virus envelope protein and its complex with a flavivirus broadly protective antibody. Cell Host Microbe 19, 696-704 (2016)

36. Cherrier, M. V. et al. Structural basis for the preferential recognition of immature flaviviruses by a fusion-loop antibody. EMBO J. 28 , 3269-3276 (2009).

37. Larocca, R. A. et al. Vaccine protection against Zika virus from Brazil. Nature 536, 474-478 (2016)

38. Abbink, P. et al. Durability and correlates of vaccine protection against Zika virus in rhesus monkeys. Sci. Transl. Med. 9, eaao4163 (2017).

39. Smith, S. A. et al. Dengue virus prM-specific human monoclonal antibodies with virus replication-enhancing properties recognize a single immunodominant antigenic site. J. Virol. 90, 780-789 (2016).

40. Tsai, W. Y. et al. High-avidity and potently neutralizing cross-reactive human monoclonal antibodies derived from secondary dengue virus infection. J. Virol. 87, 12562-12575 (2013).

41. Lazear, H. M. et al. A mouse model of Zika virus pathogenesis. Cell Host Microbe 19, 720-730 (2016)

42. Pierson, T. C. et al. The stoichiometry of antibody-mediated neutralization and enhancement of West Nile virus infection. Cell Host Microbe 1, 135-145 (2007)

43. Crill, W. D. \& Chang, G. J. Localization and characterization of flavivirus envelope glycoprotein cross-reactive epitopes. J. Virol. 78, 13975-13986 (2004).

44. Bennett, K. M. et al. Hybrid flagellin as a T cell independent vaccine scaffold. BMC Biotechnol. 15, 71 (2015).

45. Deng, Y. Q. et al. A broadly flavivirus cross-neutralizing monoclonal antibody that recognizes a novel epitope within the fusion loop of E protein. PLoS ONE 6, e16059 (2011).

46. Oliphant, T. et al. Antibody recognition and neutralization determinants on domains I and II of West Nile Virus envelope protein. J. Virol. 80, 12149-12159 (2006).

47. van der Linden, V. et al. Description of 13 infants born during October 2015-January 2016 with congenital Zika virus infection without microcephaly at birth-Brazil. MMWR Morb. Mortal. Wkly Rep. 65, 1343-1348 (2016).

48. Fernandez, E. et al. Human antibodies to the dengue virus E-dimer epitope have therapeutic activity against Zika virus infection. Nat. Immunol. 18, 1261-1269 (2017).

49. Rouvinski, A. et al. Covalently linked dengue virus envelope glycoprotein dimers reduce exposure of the immunodominant fusion loop epitope. Nat. Commun. 8, 15411 (2017).

50. Crill, W. D. et al. Sculpting humoral immunity through dengue vaccination to enhance protective immunity. Front. Immunol. 3, 334 (2012).

Publisher's note Springer Nature remains neutral with regard to jurisdictional claims in published maps and institutional affiliations.

(c) The Author(s), under exclusive licence to Springer Nature America, Inc. 2021 


\section{Methods}

Cells, viruses and animals. BHK-21 cells (ATCC catalog no. CCL-10), HEK293 cells (ATCC catalog no. CRL-1573), human embryonic kidney cells 293 T (HEK293T, ATCC catalog no. CRL-3216) and African green monkey kidney epithelial cells (Vero cells; ATCC catalog no. CCL-81) were all maintained in complete DMEM (Gibco) supplemented with $10 \%$ fetal bovine serum (FBS; Gibco) and incubated at $37^{\circ} \mathrm{C}$ under $5 \% \mathrm{CO}_{2}$. K562 cells (ATCC catalog no. CCL-243) were maintained in RPMI- 1640 medium (Gibco) containing $10 \% \mathrm{FBS}$ at $37^{\circ} \mathrm{C}$ under $5 \% \mathrm{CO}_{2}$. Aedes albopictus C6/36 cells (ATCC, catalog no. CRL-166) were cultured in RPMI- 1640 medium containing $10 \%$ FBS at $28^{\circ} \mathrm{C}$.

ZIKV-SMGC-1 was isolated in our laboratory previously ${ }^{3}$. DENV1 was isolated from an infected patient in Shenzhen Third People's Hospital, Shenzhen, China. DENV2 strain New Guinea C (NGC strain) (GenBank accession no. KM204118.1) was kindly provided by J. An (Capital Medical University, Beijing, China). DENV3 strain YN02 (GenBank accession no. KF824903) and DENV4 strain GZB5 (GenBank accession no. AF289029) were kindly provided by C.-F. Qin (Beijing Institute of Microbiology and Epidemiology, Beijing, China). ZIKV, DENV1, DENV2, DENV3 and DENV4 were propagated in C6/36 cells and titrated by a focus-forming assay on BHK-21 cells.

BALB/c mice were purchased from Beijing Vital River Laboratory Animal Technology Company (licensed by Charles River), and housed in specific-pathogen-free (SPF) mouse facilities in the Institute of Microbiology, Chinese Academy of Science (IMCAS). Ifnar $1^{-/-}$mice were purchased from the Institute of Laboratory Animal Science, Chinese Academy of Medical Sciences and Peking Union Medical College (CAMS\&PUMC), and bred in the Laboratory Animal Center, Chinese Center for Disease Control and Prevention (China CDC). AG6 mice deficient in both IFNAR1 and IFNGR were generated by Q. Leng (Institut Pasteur of Shanghai, CAS, China) from C57BL/6 background Ifngr ${ }^{-/}$ mice (Jackson laboratories, catalog no. 003288) and Ifnar $1^{-/}$A129 mice (B\&G Company). They were bred in the Laboratory Animal Center, China CDC. All animals were allowed free access to water and a standard chow diet and provided with a 12 -h light and dark cycle (temperature, $20-25^{\circ} \mathrm{C}$; humidity, $40-70 \%$ ).

Ethics statement. All animal experiments were approved by the Committee on the Ethics of Animal Experiments of the IMCAS, and conducted in compliance with the recommendations in the Guide for the Care and Use of Laboratory Animals of the IMCAS Ethics Committee.

\section{Construction and production of recombinant chimpanzee adenovirus.} The recombinant chimpanzee type 7 adenovirus (AdC7) encoding Japanese encephalitis virus signal peptide (JEVsp) and full-length membrane and envelope (M/E, defined as amino acids 216-794 of the ZIKV polyprotein) genes from ZIKV-FSS13025 (GenBank accession no. JN860885.1) was generated in our laboratory previously ${ }^{34}$. The prM/E-WT construct contains the same signal peptide (JEVsp) and full-length precursor membrane and envelope (prM/E, defined as amino acids 123-794 of the ZIKV polyprotein). Plasmid containing JEVsp-M/E-WT was used as the template to generate MutA (D98N, N103T, G106F, L107E, F108W), MutB (D98N, N103T, G106F, L107K, F108W) and MutC (D98N, N103T, G106L, L107E, F108W). The prM/E-WT, M/E-MutB and M/E-MutC cassettes were cloned into $\mathrm{pAdC}$, forming recombinant adenovirus genomes, which were linearized and transfected into HEK293 cells to rescue the recombinant adenovirus that was then further propagated and purified by cesium chloride density gradient centrifugation as previously described ${ }^{34}$.

Protein expression and purification of ZIKV/DENV-sE proteins. The coding sequence for ectodomain residues of E proteins from ZIKV (1-409), DENV1 (1-400), DENV2 (1-398), DENV3 (1-400) and DENV4 (1-400) were codon optimized and cloned into pET21a vector (Novagen) with NdeI and XhoI restriction sites. The coding sequence for the ectodomain of $\mathrm{E}-\mathrm{MutC}$ was generated by introducing mutations D98N, N103T, G106L, L107E and F108W into the WT-ZIKV-E construct. sE proteins were expressed in Escherichia coli strain BL21 (DE3) as inclusion bodies and then refolded in vitro using a previously described method $^{35}$, with some modifications. Briefly, aliquots of inclusion body were diluted dropwise with stirring into refolding buffer containing $100 \mathrm{mM}$ Tris ( $\mathrm{pH} 8.0$ ), $400 \mathrm{mM}$ L-Arg HCl, $2 \mathrm{mM}$ EDTA, $5 \mathrm{mM}$ reduced glutathione, $0.5 \mathrm{mM}$ oxidized glutathione and $10 \%$ glycerol. The refolded proteins were concentrated using an Amicon 400 concentrator with $30 \mathrm{kDa}$ cutoff membrane and then adjusted to $20 \mathrm{mM}$ Tris (pH 8.0), $50 \mathrm{mM} \mathrm{NaCl}$ and $5 \%$ glycerol. Next, sE proteins were further purified using the ÄKTA Pure System (GE Healthcare) by gel filtration on a HiLoad Superdex 200 prep grade column (GE Healthcare)

Antibody expression and purification. The human monoclonal antibodies (mAbs) Z6, Z3L1, Z20, Z23 and the murine mAbs ZWT.1-10, ZMutB.1-8 and ZMutC.1-13 were expressed and purified as previously described ${ }^{31}$. Briefly, HEK293T cells were cotransfected with pCAGGS plasmids containing coding sequences for Ig heavy chain and light chain. The cell culture was collected on days 3 and 7 post-transfection. The supernatant was mixed with one volume of buffer containing $20 \mathrm{mM}$ sodium phosphate, $\mathrm{pH} 7.0$, and filtered with a $0.22-\mu \mathrm{m}$ filter. The mixture was passed through the HiTrap Protein A FF (GE Healthcare) column. The bound protein was detached from the column by $0.1 \mathrm{M}$ glycine, $\mathrm{pH}$ 3.0. The elution was adjusted to neutral $\mathrm{pH}$ by adding $1 \mathrm{M}$ Tris-HCl, $\mathrm{pH} 9.0$ and further purified by gel filtration. The antibody was finally buffered with PBS, concentrated and stored at $-80^{\circ} \mathrm{C}$ before further use.

Murine mAb 2A10G6 was prepared according to a previously described method $^{35}$. Briefly, hybridoma cells producing 2A10G6 were injected into mice and the ascites were collected. 2A10G6 was further purified by HiTrap Protein G HP (GE Healthcare) with binding buffer of $20 \mathrm{mM}$ sodium phosphate ( $\mathrm{pH} 7.0)$ and elution buffer of $0.1 \mathrm{M}$ glycine acid- $\mathrm{HCl}(\mathrm{pH} 3.0)$. Protein was concentrated and stored at $-80^{\circ} \mathrm{C}$ until use.

To generated the fragment of antibody-binding (Fab), purified Z6, 2A10G6, Z3L1 or Z23 antibodies were digested with immobilized papain (Thermo Scientific) according to the manufacturer's instructions. Fab fractions were purified by HiTrap Protein A FF ( $5 \mathrm{ml}$, GE Healthcare) and gel filtration.

$\mathrm{Z} 3 \mathrm{~L} 1$ single-chain variable fragment $(\mathrm{scFv})$ was constructed as $\mathrm{V}_{\mathrm{L}}$ (GGGS)4- $\mathrm{V}_{\mathrm{H}}$ and cloned into pET21a. The protein was expressed in E. coli (BL21) as inclusion bodies.

Neutralization assay. The neutralizing activity of mouse serum was assessed using a previously described ZIKV microneutralization (MN) assay ${ }^{34}$, with some modifications. Briefly, heat-inactivated serum was serially diluted and incubated with ZIKV-SMGC-1 (100 PFU) for $1 \mathrm{~h}$ at $37^{\circ} \mathrm{C}$. The virus-serum mixture was added to preplated Vero cell monolayers in 96-well plates. After incubation for 4 days, the culture medium was removed, and the cells were fixed with cold methanol at $-20^{\circ} \mathrm{C}$ for $20 \mathrm{~min}$ and washed with PBS with $0.05 \%$ (v/v) Tween-20 (PBST). The following steps were all performed at room temperature (RT). The cells were blocked with $3 \%$ skim milk for $30 \mathrm{~min}$ and then stained for $2 \mathrm{~h}$ with human anti-ZIKV-E mAb Z6, which was diluted to $4 \mu \mathrm{g} \mathrm{ml}^{-1}$. Cells were washed with PBST, followed by incubation for $2 \mathrm{~h}$ with goat anti-human IgG-HRP (Proteintech), diluted 1:1,500. Plates were then washed and developed with $3,3^{\prime}, 5,5^{\prime}$-tetramethylbenzidine (TMB) substrate for $20 \mathrm{~min}$. The reaction was stopped with $2 \mathrm{M}$ hydrochloric acid, and the absorbance was measured at $450 \mathrm{~nm}$ using a microplate reader (PerkinElmer). Neutralization titers, $\mathrm{MN}_{50}$, were determined by fitting nonlinear regression curves using GraphPad Prism (v.6 and v.9.0.1) as the reciprocal of the serum dilution required for $50 \%$ neutralization of infection. $\mathrm{MN}_{50}$ titers calculated as $<20$ were reported as half the limit of detection (LOD).

Epitope detection for ZIKV-E by flow cytometry. HEK293T cells were transfected with pCAGGS plasmids encoding WT or mutated ZIKV-M/E proteins, or infected with AdC7 viruses encoding WT or mutated ZIKV-M/E proteins. After $48 \mathrm{~h}$, the cells were digested by trypsin and collected, followed by incubation in Fixation and Permeabilization solution buffer (BD, catalog no. 554722) for $20 \mathrm{~min}$ at $4^{\circ} \mathrm{C}$. The cells were then divided into several aliquots and each was stained with $\mathrm{mAb}$ (Z6, 2A10G6, Z3L1, Z23 or Z20) for $1 \mathrm{~h}$ at $4^{\circ} \mathrm{C}$. The cells were then washed twice with $1 \times$ Perm/Wash buffer and stained with Goat Anti-Human IgG-fluorescein isothiocyanate (FITC) (Proteintech) for $1 \mathrm{~h}$ at $4^{\circ} \mathrm{C}$. After washing twice with $1 \times$ Perm/Wash buffer, the cells were resuspended in PBS. Subsequently, FITC-positive HEK293T cells were detected by FACSCanton flow cytometer (BD Bioscience). The data were further analyzed by Flowjo v.7.6.1.

Capture ELISA. Cell supernatants were collected from HEK293T cells 3 days after plasmid transfection or 2 days after infection. Purified mouse anti-ZIKV IgG were plated at $500 \mathrm{ng}$ per well in 96-well ELISA plates overnight at $4^{\circ} \mathrm{C}$. Plates were blocked with $5 \%$ skimmed milk in room temperature for $30 \mathrm{~min} ; 200 \mu \mathrm{l}$ cell supernatant was added to each well overnight at $4^{\circ} \mathrm{C}$. Antibody Z3L1 conjugated with horseradish peroxidase (HRP) was used to trap the antigen at a concentration of $1 \mu \mathrm{g} \mathrm{ml}^{-1}$ at RT for $3 \mathrm{~h}$. TMB chromogen solution was then used for color development reaction, which was stopped by addition of $2 \mathrm{M} \mathrm{HCl}$. Reaction color changes were quantified at $450 \mathrm{~nm}$ in a microplate reader (PerkinElmer).

Immunization. AdC7 vaccines expressing ZIKV-M/E-WT, -prM/E-WT, -M/E-MutB or $-\mathrm{M} / \mathrm{E}-\mathrm{MutC}$ were diluted in PBS. BALB/c mice or Ifnar $1^{-/-}$mice were immunized with vaccine through the intramuscular (i.m.) route. Equivalent volume of PBS was injected as a sham control. The blood samples were collected postvaccination. The sera isolated were used for determination of antibody titer or ADE activity.

Animal protection against virus challenge. To evaluate the protective efficacy of ZIKV vaccines against lethal ZIKV challenge, immunized Ifnar ${ }^{-1-}$ mice were infected with ZIKV-SMGC-1 via i.p. injection. After ZIKV challenge, the animals were monitored daily for clinical signs of disease. Sera were collected to determine viral loads by quantitative reverse transcription-polymerase chain reaction (qRTPCR). Mice that had lost $25 \%$ of initial body weight were euthanized on animal welfare grounds.

To measure virus burdens in targeted tissues, immunized Ifnar $1^{-/-}$mice were i.p. challenged with ZIKV-SMGC-1. Mice were euthanized and necropsied. Organs were removed, weighed and homogenized in PBS with a tissue grinder. Samples were further centrifuged, and the supernatants were stored at $-80^{\circ} \mathrm{C}$ until virus titration by qRT-PCR. 
qRT-PCR. Virus RNA was isolated from $200 \mu$ l supernatants of homogenized tissues or $50 \mu \mathrm{l}$ of mouse serum using a GenePure Plus nucleic acid extraction instrument (Hangzhou Bioer Technology). ZIKV-specific qRT-PCR assays were performed using a FastKing One Step Probe RT-qPCR kit (Tiangen Biotech) on a CFX96 Touch real-time PCR detection system (Bio-Rad) according to the manufacturer's protocol. Primers and probe were designed to amplify a region of the NS3 gene from ZIKV-FSS13025 and ZIKV-SMGC-1, with sequences as follows: forward primer, TTGGCTGGCCTATCAGGTTG; reverse primer, CACCTCGGTTTGAGCACTCT; the probe

\section{FAM-CCACACCTCTGCCGGCACAC-TAMRA (where FAM is}

6-carboxyfluorescein, and TAMRA is 6-carboxytetramethylrhodamine). Viral loads were expressed on a $\log _{10}$ scale as viral copies per gram or copies per milliliter after calculation with a standard curve. Viral copy numbers below the LOD were set as half of the LOD.

ELISA. Binding properties of murine sera and the isolated mAbs to ZIKV-sE (monomer), ZIKV-sE (dimer), ZIKV-sE-MutC or DENV-sE (serotype 1-4) were determined by ELISA. First, 96-well plates (catalog no. 3590; Corning) were coated overnight with $3 \mu \mathrm{g} \mathrm{ml}^{-1}$ of ZIKV/DENV-E or -E-mutant in $0.05 \mathrm{M}$ carbonate-bicarbonate buffer, pH 9.6, and blocked in 5\% skim milk in PBS. Sera were serially diluted and added to each well. For mAbs expressed from HEK293T cells, culture supernatant was added. Plates were incubated with goat anti-mouse IgG-HRP antibody (murine serum and mAbs isolated from mice) or goat anti-human IgG-HRP antibody (mAb Z6, Z3L1 and Z23), and subsequently developed with TMB substrate. Reactions were stopped with $2 \mathrm{M}$ hydrochloric acid, and the absorbance was measured at $450 \mathrm{~nm}$ using a microplate reader (PerkinElmer). The endpoint titers were defined as the highest reciprocal dilution of serum to give an absorbance greater than fivefold background values.

In vitro evaluation of ADE of virus infection. Heat-inactivated serum was serially diluted with RPMI-1640 medium supplemented with 1\% FBS and incubated with ZIKV or DENV1, 2, 3 or 4 for $1 \mathrm{~h}$ at $37^{\circ} \mathrm{C}$. The serum-virus mixture was then mixed with $3 \times 10^{4} \mathrm{~K} 562$ cells and incubated for $2 \mathrm{~h}$ at $37^{\circ} \mathrm{C}$. RPMI- 1640 medium supplemented with $2 \%$ FBS were then added. After incubation for 4 days, the K562 cells were centrifuged and washed once with PBS, followed by incubation with Fixation and Permeabilization solution (BD, catalog no. 554722) for $20 \mathrm{~min}$ at $4{ }^{\circ} \mathrm{C}$. The cells were washed twice with $1 \times$ Perm/Wash buffer $(\mathrm{BD}, 554723)$ and stained with FITC-conjugated human mAb Z6 for $1 \mathrm{~h}$ at $4^{\circ} \mathrm{C}$. After washing twice with $1 \times$ Perm/Wash buffer, the cells were resuspended in PBS. Virus infected K562 cells were detected by FACSCanton flow cytometer (BD Bioscience) and further analyzed by Flowjo v.7.6.1.

In vivo ADE of DENV2 evaluation. AG6 mice were used as the infection model. BALB/c mice were immunized with vaccines or sham as sera donor. At 4 weeks postvaccination, blood samples were collected, and sera were isolated, heat-inactivated, mixed together for each vaccine group, aliquoted and stored at $-80^{\circ} \mathrm{C}$ until use. Before adoptive transfer, the sera were first diluted tenfold with PBS. AG6 mice received diluted sera by passive transfer. AG6 mice were challenged with DENV2-NGC and monitored daily for survival and weight loss postchallenge. Mice that had lost $25 \%$ of initial body weight were euthanized on animal welfare grounds

Mouse pregnancy experiment. Female Ifnar $1^{-/-}$mice were vaccinated with vaccines or sham. Serum samples were collected on day 28 , heat inactivated $\left(56^{\circ} \mathrm{C}\right.$ for $30 \mathrm{~min}$ ) for detection of ZIKV-E-specific IgG and $\mathrm{MN}_{50}$. Ifnar1 ${ }^{-/-}$female mice were then mated with Ifnar $1^{-/-}$males in individual cages. The vaginal plug was checked daily. Once the plug was identified, the mice were defined at starting day embryotic stage (d_e0.5). The mice were infected with ZIKV-SMGC-1 at d_e5.5. All animals were sacrificed at d_e13.5. Blood, brain, spleen of maternal mice and placentas and heads of fetuses were harvested for measuring viral loads by qRT-PCR

SPR assay. SPR binding experiments were carried out using a BIAcore 8000 device (GE Healthcare) at RT. The buffers for all proteins used for kinetic analyses were exchanged for BIAcore buffer consisting of $10 \mathrm{mM}$ HEPES (pH 7.4), $150 \mathrm{mM} \mathrm{NaCl}$, $3 \mathrm{mM}$ EDTA and $0.005 \%$ (v/v) Tween-20 by gel filtration. Purified ZIKV-sE or ZIKV-sE-MutC proteins were immobilized on a CM5 chip with the standard EDC/ NHS coupling method at about 6,000 response units (RU). Serial dilutions of Fabs were prepared and used to flow over the chip surface. Data were collected over time. After each cycle, the sensor surface was regenerated via a short treatment using $30 \mathrm{mM} \mathrm{NaOH}(\mathrm{Z} 6 \mathrm{mAb})$ or $3 \mathrm{M} \mathrm{MgCl}_{2}$ (2A10G6 antibody). The apparent equilibrium dissociation constants (apparent binding affinity, $K_{\mathrm{D}}$ ) for each sE-antibody were calculated using BIAcore 8000 analysis software (BIAevaluation v.4.1). Each set of equilibrium binding responses was fitted to the single binding site model.

Isolation of antigen-specific $\mathbf{B}_{\mathrm{GC}}$ cells. Single-cell suspensions were obtained from the lymph nodes of immunized mice. The cells from each group of mice were pooled together. Antibodies used for staining were anti-mouse GL-7-FITC,
CD138-PE, CD38-PE/Cy7, CD93-APC, B220-BV421 and IgD-BV510. All antibodies were used according to manufacturer's instructions. To stain the ZIKV-E-binding B cells, ZIKV-sE monomer (described above) and dimer (a kind gift from R. Gong, Wuhan Institute of Virology, CAS, China $)^{51}$ were used as bait by biotinylation via Biotin-Protein Ligase/BirA Enzyme (GeneCopoeia). BV711Streptavidin was used to probe the biotinylated-ZIKV-E proteins. Antigen-specific $\mathrm{B}_{\mathrm{GC}}$ gated as $\mathrm{GL}-7^{+}, \mathrm{B} 220^{\text {hi }}, \mathrm{CD} 38^{\text {lo }}, \mathrm{IgD}^{-}, \mathrm{CD}^{-}, \mathrm{CD} 138^{-}$and $\mathrm{ZIKV}-\mathrm{E}^{+}$were sorted using a BD FACSAria II flow cytometer (BD Biosciences).

Single-cell library construction and sequencing. Samples for single BCR V(D)J clonotype were processed using a Chromium Single Cell 5' Library and Gel Bead Kit following the manufacturer's user guide (10x Genomics). After FACS sorting, cells were centrifuged, resuspended in 3\% FBS (Sigma-Aldrich)/phosphate buffer solution (Thermo Fisher Scientific) and subjected to cell quality control using a cell counter. All processed B cells had cell viability $>70 \%$. After determining cell density, cells were injected into three channels, aiming to achieve around 1,000-3,000 cells per channel. Gel beads-in-emulsion (GEMs) were formed in channels of a chip in the 10x Chromium instrument, and then collected in an Eppendorf plate for the GEM-reverse transcription (GEM-RT) reaction. After GEM clean up, GEM-RT products were subjected to two rounds of 9 and 15 PCR cycles using custom primers, followed by SPRIselect (Beckman Coulter) bead clean up. Single-cell BCR V(D)J libraries were prepared following the manufacturer's user guide, profiled using the Bioanalyzer High Sensitivity DNA kit (Agilent Technologies) and quantified with a Kapa Library Quantification Kit (Kapa Biosystems). Libraries were sequenced by paired-end sequencing $(2 \times 150 \mathrm{bp})$ on an Illumina NovaSeq (Illumina). BCL data were converted to demultiplexed FASTQ files using Illumina bcl2fastq v.2.20.

Cellranger V(D)J data processing. We retained the first 26 bases for read 1 covering the 16-nt cell barcode and 10-nt unique molecular identifier (UMI). The FASTQ files were analyzed subsequently. The Cell Ranger Single-Cell Software Suite (v.3.1.0) was used to perform barcode processing and single-cell V(D)J analysis. The FASTQ files were processed using the Cell Ranger V(D)J pipeline. First, reads were filtered for valid cell barcodes and UMIs. Cell barcodes with 1-Hamming-distance from a list of known barcodes were considered. A UMI with 1-Hamming-distance from another UMI with more reads for a same barcode was corrected to this UMI with more reads. The filtered reads were used to assembly contigs by alignment with the GRCm $38 \mathrm{~V}(\mathrm{D}) \mathrm{J}$ reference genome, then defined alignments of V, D and J segments to a contig, identifying CDR3 sequences, and, from these data, determine whether a contig is productive, meaning that it is likely to correspond to a functional B cell receptor. The $\mathrm{V}(\mathrm{D}) \mathrm{J}$ sequences and clonotypes from single-cell $5^{\prime}$ data were analyzed, searched and visualized using the LoupeTM $\mathrm{V}(\mathrm{D}) \mathrm{J}$ browser v.3.0.0. Sequences were further annotated and analyzed using IgBLAST v1.6.1 (ref. ${ }^{52}$ ) to identify variable region gene segments and somatic mutations.

Crystallization, data collection and structure determination. For the ZIKV-sE/ Z6 complex, ZIKV-sE was mixed with Z6-Fab at a stoichiometry of 1.3:1 and incubated on ice for $2 \mathrm{~h}$. The mixture was then subjected to gel filtration. The survival peak containing the indicated $\mathrm{Fab}$ and $\mathrm{sE}$ was pooled, buffered in $20 \mathrm{mM}$ Tris, $50 \mathrm{mM} \mathrm{NaCl}$ and $5 \%$ glycerol (pH 8.0). It was concentrated to 10 or $5 \mathrm{mg} \mathrm{m}^{-1}$ for crystallization.

To obtain ZIKV-sE-MutC/Z3L1 complex, the inclusion bodies of ZIKV-sE-MutC and Z3L1-scFv were diluted into refolding buffer containing $100 \mathrm{mM}$ Tris ( $\mathrm{pH} 8.0$ ), $400 \mathrm{mM} \mathrm{L}-\mathrm{Arg} \mathrm{HCl}, 2 \mathrm{mM}$ EDTA, $5 \mathrm{mM}$ reduced glutathione, $0.5 \mathrm{mM}$ oxidized glutathione and $10 \%$ glycerol. After $12 \mathrm{~h}$ of slow stirring at $4{ }^{\circ} \mathrm{C}$, the refolded ZIKV-sE-MutC/Z3L1-scFv complex was concentrated and further purified in an ÄKTA Pure System (GE Healthcare) by gel filtration on a HiLoadTM SuperdexTM 200 preparation grade column (GE Healthcare). The survival peak containing the indicated ZIKV-sE-MutC/Z3L1-scFv was pooled and further polished using an ion exchange column (Source 15Q; GE Healthcare). The indicated complex was further buffered in $20 \mathrm{mM}$ Tris, $50 \mathrm{mM} \mathrm{NaCl}$ and $5 \%$ glycerol (pH 8.0), and concentrated to 10 or $5 \mathrm{mg} \mathrm{ml}^{-1}$ for crystallization.

Crystallization trials were performed in sitting drops of $600 \mathrm{nl}$. All crystals were obtained by mixing equal volumes of protein and reservoir solution, using a Mosquito LCP robot (TTP LabTech). Diffraction-quality crystals of ZIKV-sE/ Z6-Fab were obtained at $18^{\circ} \mathrm{C}$ in condition consisting of $0.1 \mathrm{M}$ amino acids, $0.1 \mathrm{M}$ buffer system 3 at pH 8.5 (Morpheus MD1-46) and 30\% v/v Precipitant Mix 3 (Morpheus MD1-46). Diffraction-quality crystals of ZIKV-sE-MutC/Z3L1-scFv were obtained at $18^{\circ} \mathrm{C}$ in conditions consisting of $0.12 \mathrm{M}$ ethylene glycol, $0.1 \mathrm{M}$ buffer system 3 (Morpheus MD1-46) at pH 8.5 and 30\% v/v Precipitant Mix 2 (Morpheus MD1-46).

All diffraction data were collected at the Shanghai Synchrotron Radiation Facility and processed with HKL2000 (ref. ${ }^{53}$ ). The ZIKV-sE/Z6-Fab dataset was collected at BL17U1, whereas the ZIKV-sE-MutC/Z3L1-scFv dataset was collected at BL19U1. The complex structure was solved by the molecular replacement module of $\mathrm{Phaser}^{54}$ from the CCP4 program suite ${ }^{55}$, with the previously reported structure of sE from ZIKV (PDB 5JHM), ZIKV-sE/2A10G6-Fab (PDB: 5JHL) and 
ZIKV-sE/Z3L1-Fab (PDB 5GZN) as the search models. Restrained rigid-body refinement with REFMAC5 (CCP4 suite) and manual model building with Coot $^{56}$ were then performed. Further rounds of refinement were then done with phenix. refine ${ }^{57}$. During model building and refinement, the program of MolProbity ${ }^{58}$ was used to validate the stereochemistry of the structure. Structural data were analyzed by PyMOL and the figures were generated using Chimera ${ }^{59}$.

Statistical analysis. GraphPad Prism v.6 or v.9.01 software was used for data analysis. Data are represented as mean \pm s.e.m. Statistical significance was determined by unpaired, two-sided Student's $t$-test for two-group comparisons, one-way analysis of variance (ANOVA) for comparisons of more than two groups, two-way ANOVA for comparisons of more than two groups with two or more items, or log-rank test for survival experiments. $P$ values $<0.05$ were considered to be statistically significant, where ${ }^{\star} P<0.05,{ }^{\star *} P<0.01,{ }^{\star * *} P<0.001$ and ${ }_{* * * *} P<0.0001$

Reporting Summary. Further information on research design is available in the Nature Research Reporting Summary linked to this article.

\section{Data availability}

The crystal structures of ZIKV-sE-Z6 and ZIKV-sE-MutC-Z3L1 have been deposited in the Protein Data Bank (PDB) under accession codes 7BQ5 and 7BPK, respectively. The raw data for $\mathrm{scBCR}$-seq have been deposited at https://www. scidb.cn/en/datalist?tag $=1$. Source data are provided with this paper. All other data supporting the findings of this study are available within the paper or from the corresponding author upon reasonable request.

\section{References}

51. Yang, C. et al. Characterization of two engineered dimeric Zika virus envelope proteins as immunogens for neutralizing antibody selection and vaccine design. J. Biol. Chem. 294, 10638-10648 (2019).

52. Ye, J., Ma, N., Madden, T. L. \& Ostell, J. M. IgBLAST: an immunoglobulin variable domain sequence analysis tool. Nucleic Acids Res. 41, W34-W40 (2013).

53. Otwinowski, Z. \& Minor, W. Processing of X-ray diffraction data collected in oscillation mode. Methods Enzymol. 276, 307-326 (1997).

54. Read, R. J. Pushing the boundaries of molecular replacement with maximum likelihood. Acta Crystallogr. D Biol. Crystallogr. 57, 1373-1382 (2001).

55. Collaborative Computational Project, Number 4. The CCP4 suite: programs for protein crystallography. Acta Crystallogr. D Biol. Crystallogr. 50, 760-763 (1994).

56. Emsley, P. \& Cowtan, K. Coot: model-building tools for molecular graphics. Acta Crystallogr. D Biol. Crystallogr. 60, 2126-2132 (2004).

57. Aad, G. et al. Search for new particles in two-jet final states in $7 \mathrm{TeV}$ proton-proton collisions with the ATLAS detector at the LHC. Phys. Rev. Lett. 105, 161801 (2010).
58. Chen, V. B. et al. MolProbity: all-atom structure validation for macromolecular crystallography. Acta Crystallogr. D Biol. Crystallogr. 66, 12-21 (2010).

59. Pettersen, E. F. et al. UCSF Chimera-a visualization system for exploratory research and analysis. J. Comput. Chem. 25, 1605-1612 (2004).

\section{Acknowledgements}

We thank the staff of BL17U and BL19U beamlines at National Center for Protein Sciences Shanghai and Shanghai Synchrotron Radiation Facility (Shanghai, China) for assistance during data collection. We are grateful to J. Jia (Institute of Biophysics, Chinese Academy of Sciences (CAS), China) for technical support during BD FACSAria II manipulation. We thank Y. Chen (Institute of Biophysics, CAS, China) for technical support with BIAcore experiments. We thank D. Zhou (Institut Pasteur of Shanghai, CAS, China) for providing us with the AdC7 vector. We thank G. Cheng (Tsinghua University, Beijing, China) and Q. Leng (Institut Pasteur of Shanghai, CAS, China) for providing AG6 mice for breeding. We thank R. Gong (Wuhan Institute of Virology, CAS) for providing ZIKV-sE dimer protein. We thank Y. Chen (China CDC) for breeding of AG6 and Ifnar $1^{-1-}$ mice. We thank C. Liu (Guangxi University, China) for technical support. This work is supported by the National Science and Technology Major Project (2016YFE0205800 and 2020YFA0907100) (L.D. and G.F.G.), the National Natural Science Foundation of China (NSFC) (81991494 and 81801648) (L.D.) and the Strategic Priority Research Program of the Chinese Academy of Sciences (XDB29010202) (G.F.G). L.D. is supported by Youth Innovation Promotion Association CAS (2018113).

\section{Author contributions}

L.D., J.Y. and G.F.G. designed the experiments. L.D., K.X., J.L., Q.H., J.S., Y.H., T.Z., P.G., X.L., H.Y., K.L., Q.W., Y.C. and J.Q. performed the investigations and assays. L.D., K.X., J.Y. and G.F.G. analyzed the data. L.D. and K.X. wrote the manuscript. L.D., K.X., Q.X., Q.W., J.Y. and G.F.G. discussed and edited the manuscript.

\section{Competing interests}

G.F.G, L.D., J.Y., K.X., Y.H., Q.W., Q.H. and J.L. are listed as the coinventors for the pending patent for the ZIKV vaccines described in this study. The other authors declare no competing interests.

\section{Additional information}

Extended data is available for this paper at https://doi.org/10.1038/s41590-021-00966-6. Supplementary information The online version contains supplementary material available at https://doi.org/10.1038/s41590-021-00966-6.

Correspondence and requests for materials should be addressed to L.D., J.Y. or G.F.G. Peer review Information Nature Immunology thanks R. Rappuoli and the other, anonymous, reviewer(s) for their contribution to the peer review of this work. Peer reviewer reports are available. J. D. K. Wilson was the primary editor on this article and managed its editorial process and peer review in collaboration with the rest of the editorial team.

Reprints and permissions information is available at www.nature.com/reprints. 
a

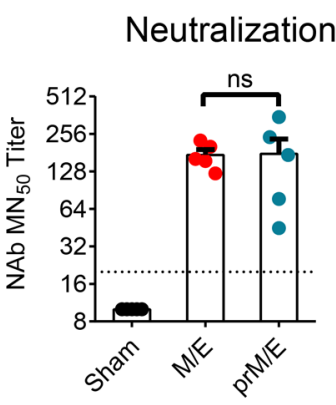

f

Z6

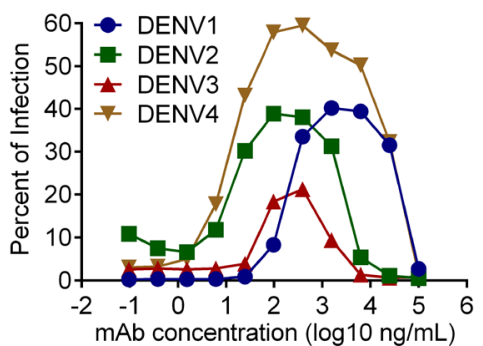

b

DENV1 enhancement

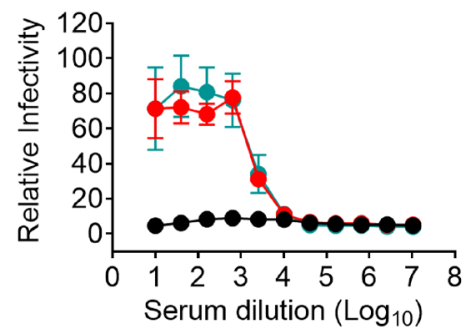

d

DENV3 enhancement

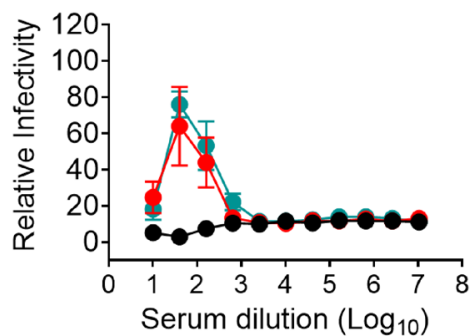

C

\section{DENV2 enhancement}

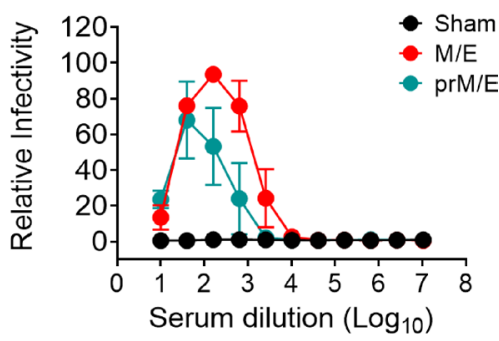

e

DENV4 enhancement

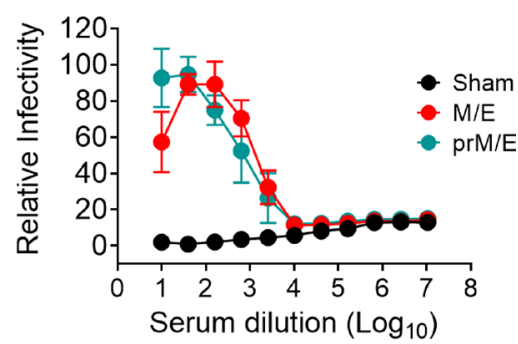

Extended Data Fig. 1 | Antibodies induced by ZIKV vaccines enhance DENV infections. a, Neutralizing activities of ZIKV vaccines. 6-8-week old female BALB/c mice ( $n=5$ biologically independent samples) were immunized with a single dose of AdC7-M/E or AdC7-prM/E (1.6 $\left.\times 10^{11} \mathrm{vp}\right)$ via i.m. route. PBS was given as a sham vaccine. Sera were collected from blood at 4 weeks postvaccination. Magnitudes of NAbs were measured as $M_{50}$ titers. Data are means \pm standard errors of means (SEM). P values were analyzed with One-way ANOVA test (ns, not significant). The dashed line indicates the LOD. b-e, ADE activities of the immune sera ( $n=3$ biologically independent samples). Enhancement curves of K562 cells infected with DENV1 (b), DENV2 (c), DENV3 (d), and DENV4 (e) in the presence of serially diluted mouse immune sera as indicated. Infected cells were quantified by flow cytometry and were normalized to the peak infectivity of FLE mAb Z6. Data are means \pm SEM. $\mathbf{f}, A D E$ activities of the human mAb Z6. Enhancement curves of K562 cells infected with DENV1, 2, 3 and 4 in the presence of serially diluted Z6 as indicated. 
a

b

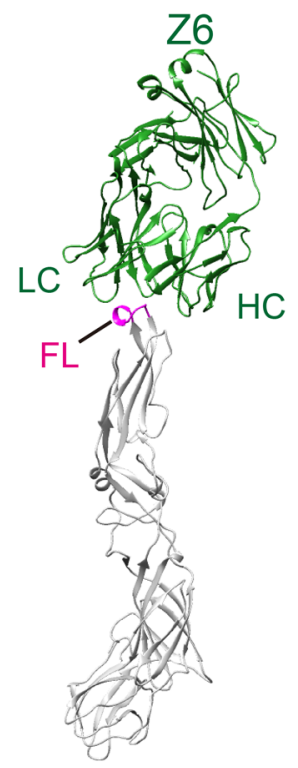

C

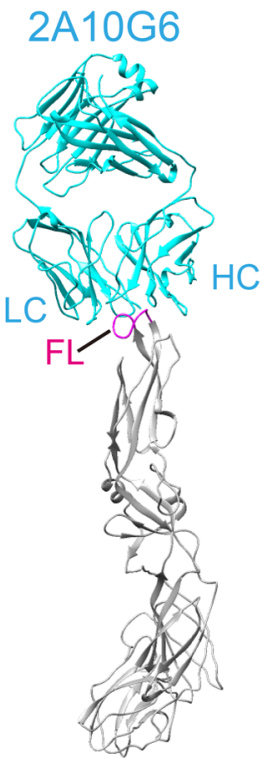

d

\section{Overlapping}

2A10G6
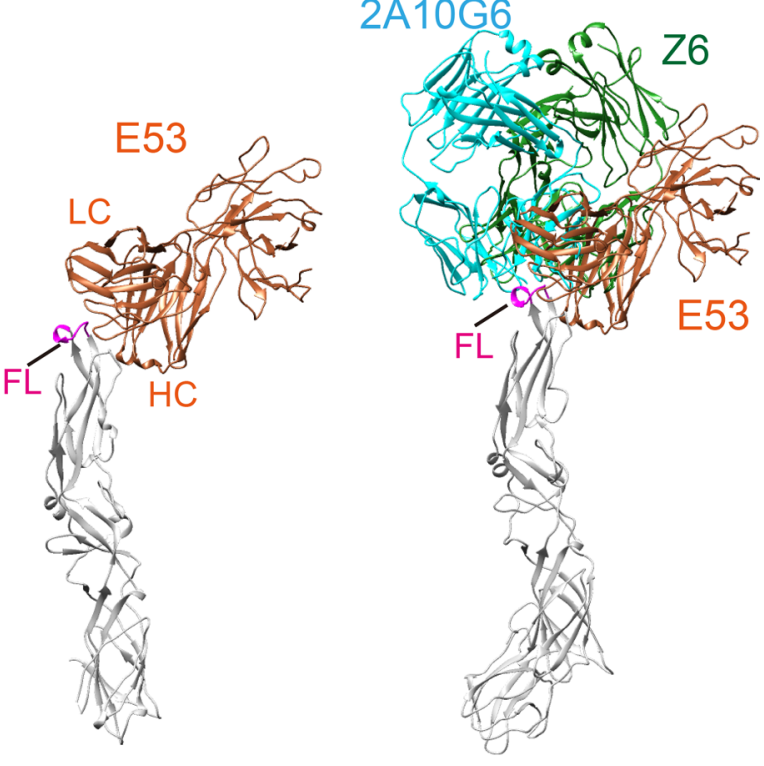

e

ZIKV sE contacts Z6

ZIKV sE contacts 2A10G6

WNV sE contacts E53

\begin{tabular}{|c|c|c|c|c|c|c|c|c|c|c|c|}
\hline $\begin{array}{l}\text { Residues in } \\
\mathrm{ZIKV} \mathrm{sE}\end{array}$ & $\begin{array}{l}\text { Heavy Chain } \\
\text { Contacts }\end{array}$ & $\begin{array}{c}\text { Light Chain } \\
\text { Contacts }\end{array}$ & $\begin{array}{c}\text { Total } \\
\text { Contacts }\end{array}$ & $\begin{array}{l}\text { Residues in } \\
\text { ZIKV sE }\end{array}$ & $\begin{array}{l}\text { Heavy Chain } \\
\text { Contacts }\end{array}$ & $\begin{array}{l}\text { Light Chain } \\
\text { Contacts }\end{array}$ & $\begin{array}{c}\text { Total } \\
\text { Contacts }\end{array}$ & $\begin{array}{l}\text { Residues in } \\
\text { WNV sE }\end{array}$ & $\begin{array}{l}\text { Heavy Chain } \\
\text { Contacts }\end{array}$ & $\begin{array}{l}\text { Light Chain } \\
\text { Contacts }\end{array}$ & $\begin{array}{c}\text { Total } \\
\text { Contacts }\end{array}$ \\
\hline P75 & & 6 & 6 & T76 & 10 & & 10 & C74 & 5 & & 5 \\
\hline $\mathrm{T} 76$ & & 15 & 15 & Q77 & 6 & & 6 & P75 & 8 & & 8 \\
\hline W101 & 59 & 25 & 84 & W101 & 59 & 24 & 83 & T76 & 36 & & 36 \\
\hline G102 & 1 & & 1 & G102 & & 6 & 6 & M77 & 32 & & 32 \\
\hline G104 & & 4 & 4 & G104 & 3 & & 3 & G78 & 8 & & 8 \\
\hline C105 & & 10 & 10 & C105 & 10 & & 10 & E79 & 16 & & 16 \\
\hline G106 & & 34 & 34 & G106 & 17 & & 17 & G104 & 10 & & 10 \\
\hline L107 & 1 & 12 & 13 & L107 & 15 & & 15 & C105 & 6 & & 6 \\
\hline F108 & 41 & 6 & 47 & G108 & 14 & 33 & 47 & G106 & 10 & 5 & 15 \\
\hline G109 & & 5 & 5 & & & & & L107 & 5 & 4 & 9 \\
\hline \multirow[t]{2}{*}{ K110 } & & 7 & 7 & & & & & G109 & 4 & & 4 \\
\hline & & & & & & & & K110 & 15 & & 15 \\
\hline Total & 102 & 124 & 226 & & 134 & 63 & 197 & & 155 & 9 & 164 \\
\hline
\end{tabular}

Extended Data Fig. 2 | Comparison of FLE mAbs bound to flavivirus-E. a, Z6 bound to ZIKV-sE. Z6 Fab is colored in green and ZIKV-sE is colored in grey. FL is highlighted in magenta. b, 2A10G6 bound to ZIKV-sE (PDB: $5 \mathrm{JHL}$ ). 2A10G6-Fab is colored in cyan and ZIKV-sE is colored in grey. FL is highlighted in magenta. c, E53 bound to WNV-sE (PDB: 3150). E53 Fab is colored in orange and ZIKV-sE is colored in grey. FL is highlighted in magenta. d, The Z6, 2A10G6 and E53 bound to E are overlapped, showing different approaching angles. e, The E residues participating in interactions of antibodies are listed. The numbers of contacts are highlighted for the main contributors in each sE. 
a

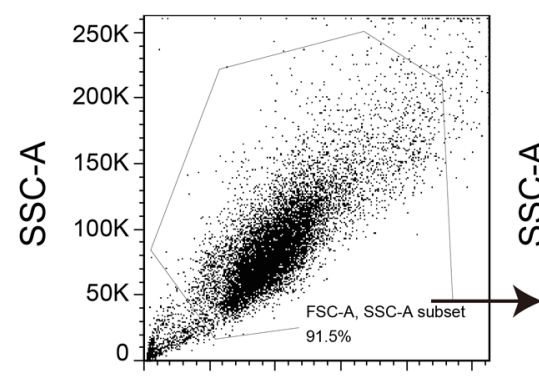

$0 \quad 50 \mathrm{~K} 100 \mathrm{~K} 150 \mathrm{~K} 200 \mathrm{~K} 250 \mathrm{~K}$

FSC-A

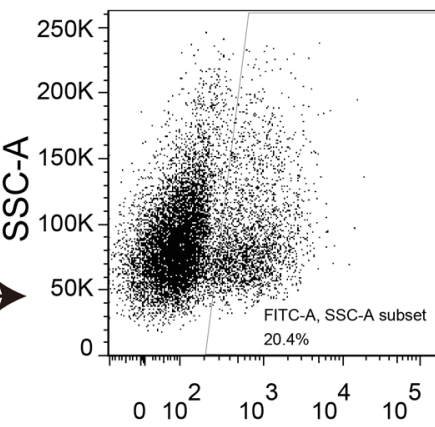

2A10G6-FITC

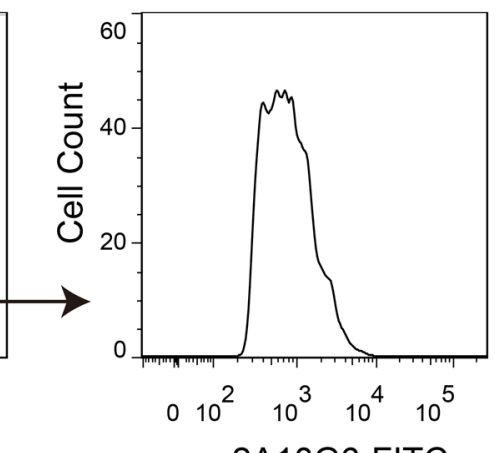

2A10G6-FITC

b

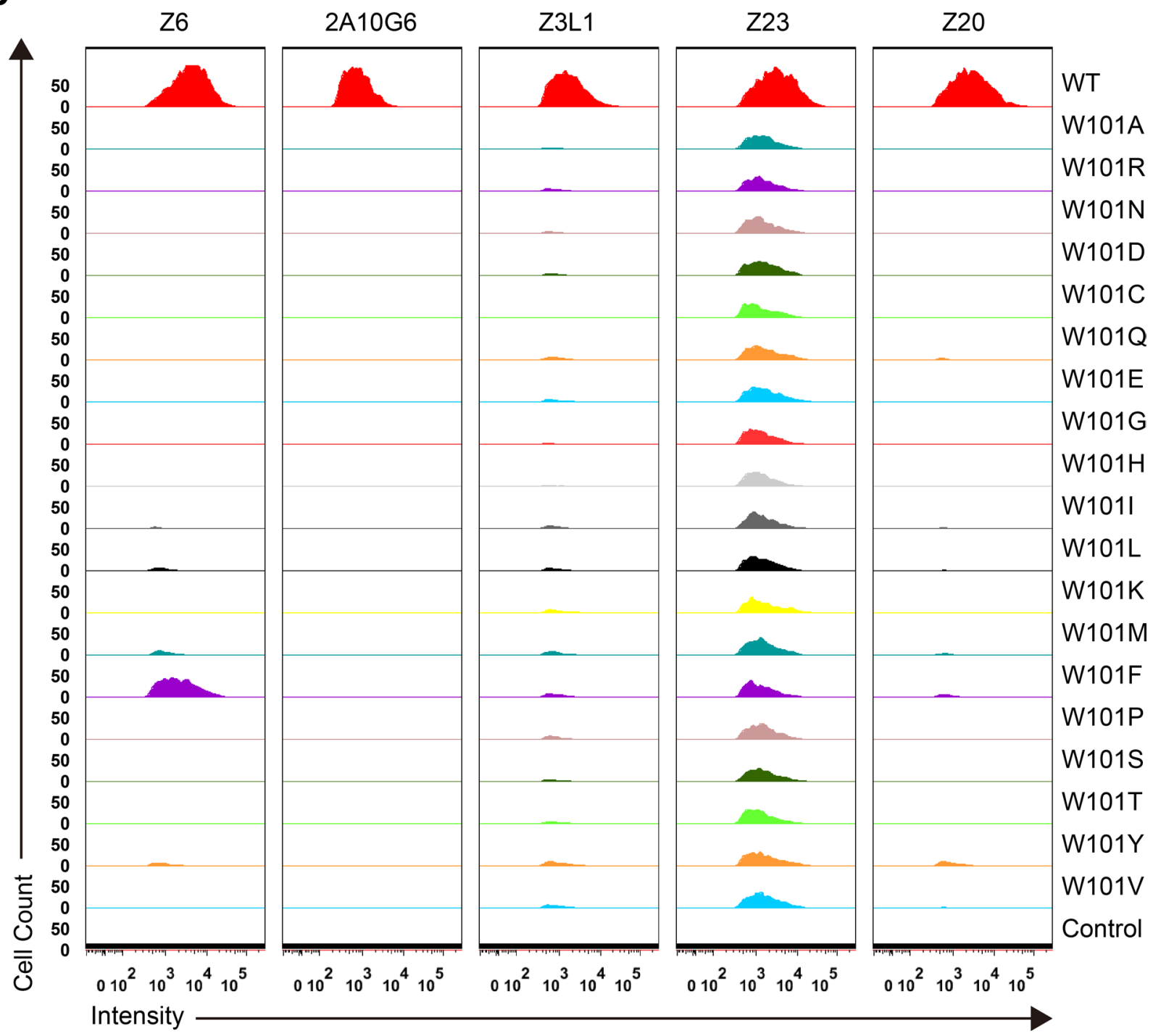

Extended Data Fig. 3 | Flow cytometry detection of antigen expression and epitope display. a, Gating strategy to monitor ZIKV antigen expression by FACS analysis, related to Fig. 1e, Extended Data Figs. 3b and 5a. b. Plasmid expressing M/E-WT and 19 E-W101 mutants as indicated were probed by FLE mAbs or neutralizing mAbs targeting DI, DII or DIII. 
a

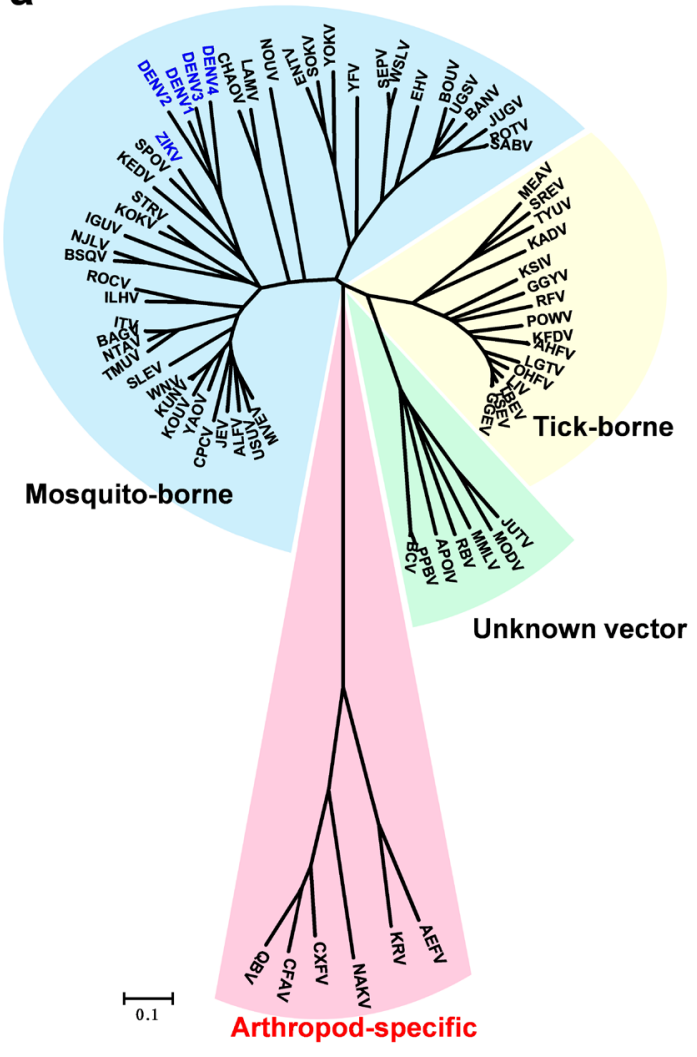

b

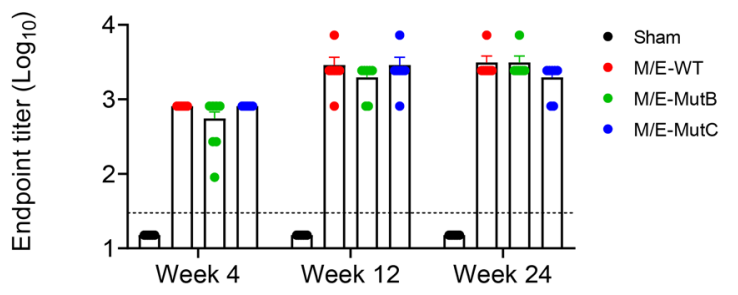

C

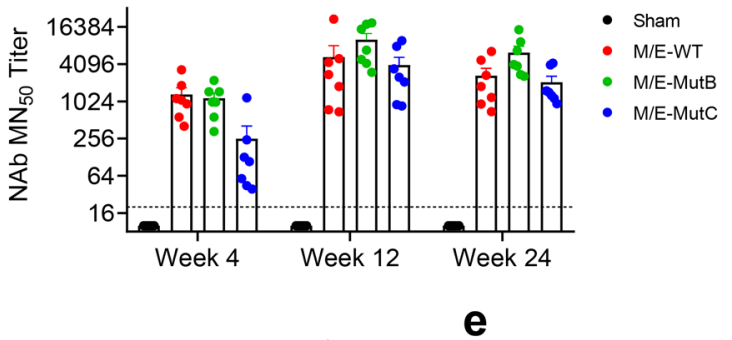

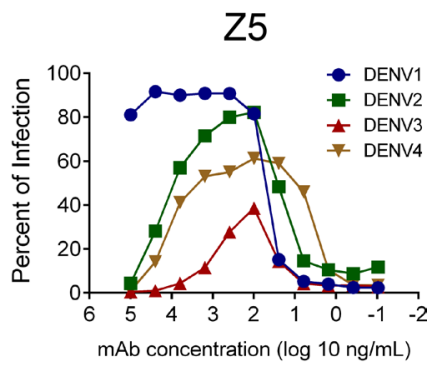

Extended Data Fig. 4 | Phylogenetic trees flaviviruses, neutralizing titers in Ifnar ${ }^{-1}$ mice and ADE activities of the human mAb Z5. a, Phylogenetic trees of 73 flaviviruses based on the amino acid sequences of the E. b-c, Groups of 5-week-old female Ifnar ${ }^{-1-}$ mice $(n=7)$ were immunized with a single dose $\left(1.6 \times 10^{11} \mathrm{vp}\right)$ of AdC7-M/E-WT, - M/E-MutB or $-\mathrm{M} / \mathrm{E}-\mathrm{MutC}$ via i.m. route. PBS was given as the sham vaccine. Sera were collected at week 4,12 and 24. Serologic binding and neutralizing antibodies were detected. Data are means \pm SEM. d, Groups of 5-week-old Female Ifnar ${ }^{-1-}$ mice $(n=8-9)$ were immunized with a single dose $\left(1.6 \times 10^{11} \mathrm{vp}\right)$ of AdC7-M/E-WT, $-M / E-M u t B$ or $-M / E-M u t C$ via i.m. route. PBS was given as the sham vaccine. Sera were collected at 4 weeks postimmunization. $M N_{50}$ titers of NAbs were measured. Results were pooled from two independent experiments (related to Fig. $2 b-e$ ). Data are means \pm SEM. P values were analyzed with two-tailed Student's t test (ns, not significant; ${ }^{*}, \mathrm{P}<0.05 ; \mathrm{P}$ values are available in source data). The dashed line indicates the LOD. e, ADE activities of the ZIKV FLE mAb Z5. Enhancement curves of K562 cells infected with DENV1, 2, 3 and 4 in the presence of serially diluted $Z 5$ as indicated. Infected cells were quantified by flow cytometry. 
a

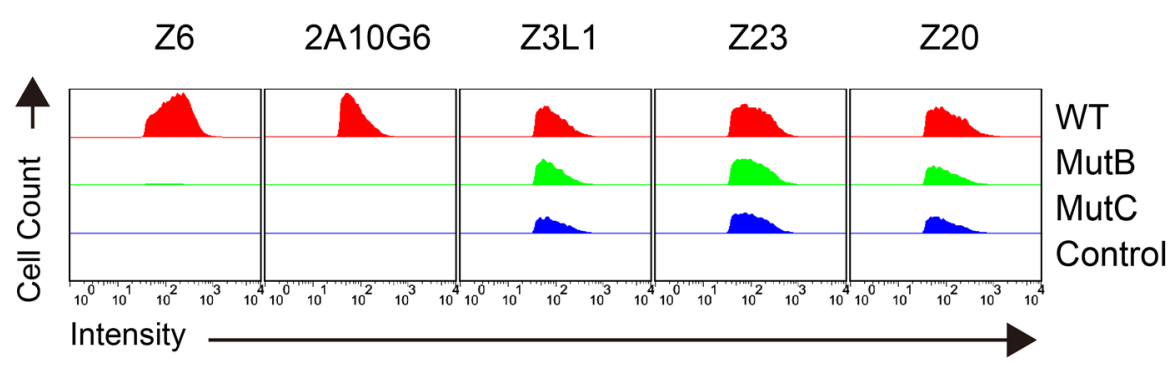

b

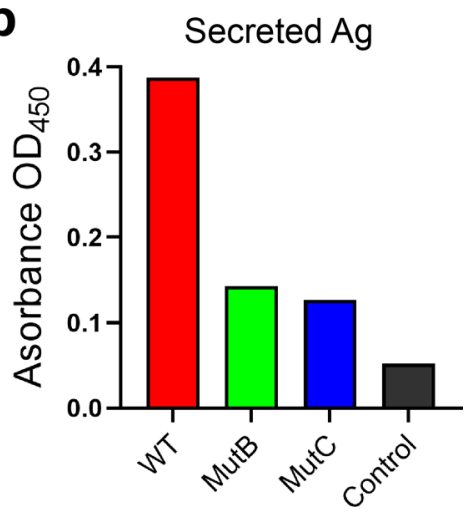

Extended Data Fig. 5 | Antigen characterization of AdC7 vectored vaccines. HEK293 cells were infected with AdC7 expressing ZIKV-M/E WT, MutB or MutC. Non-infected cells were used as the negative control. $\mathbf{a}$, Flow cytometry detection of antigen expression and epitope display probed by FLE mAbs or neutralizing mAbs targeting epitopes on DI, DII or DIII. b, Capture ELISA quantifying the secretion of ZIKV antigen from AdC7 virus infected cells. Data are means of triplicates. 
a

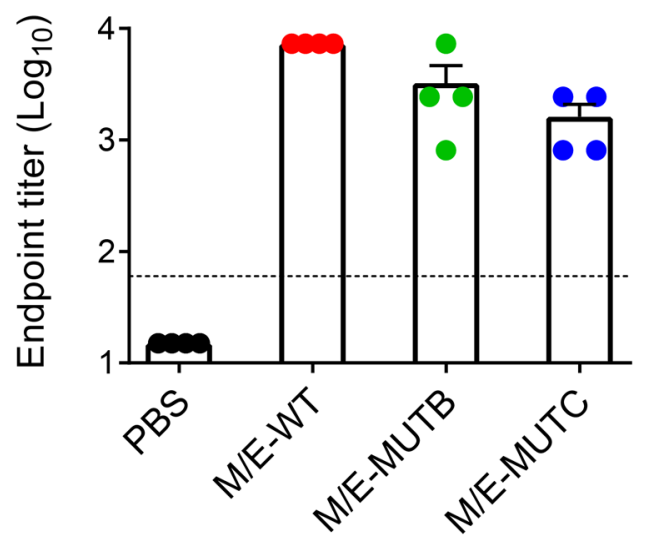

C

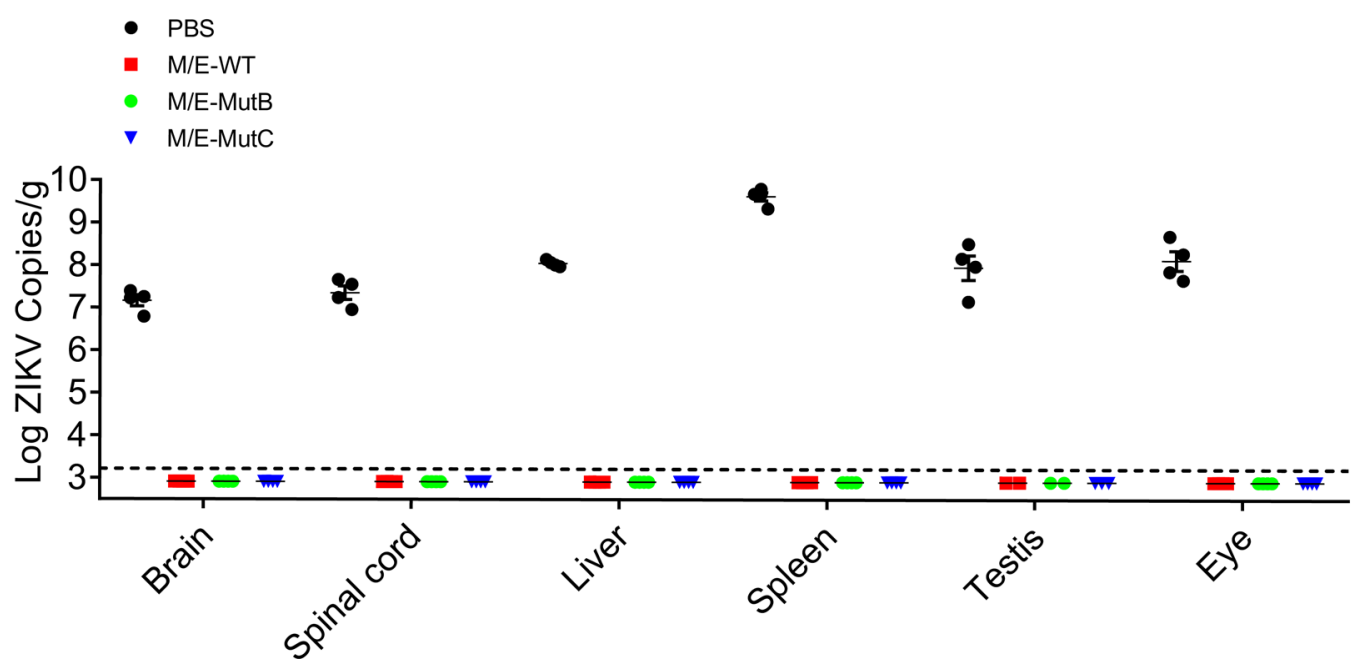

Extended Data Fig. 6 | Protective efficacy of MutB/C-based vaccine against ZIKV infection at 3 DPI. a-c, Groups of Ifnar ${ }^{-/-}$mice $(n=4)(F e m a l e: m a l e$ of 2:2 in both WT and MutB groups; 3:1 in MutC group; 4:0 in sham group) received a single immunization of 1.6 $\times 10^{11} \mathrm{vp}$ of AdC7-M/E-WT, AdC7-M/E-MutB, AdC7-M/E-MutC via the i.m. route. PBS was given as the sham vaccine. Serum samples were collected for detection of ZIKV-E-specific IgG (a) and NAb titers (b). Thirty-days after vaccination, mice were challenged with $5 \times 10^{6} \mathrm{FFU}$ of ZIKV-SMGC-1 via the i.p. route. At 3 DPI, tissues from the brain, spinal cord, testis, spleen, liver and eye were harvested for measurement of ZIKV loads (c). Data are means \pm SEM. The dashed line indicates the LOD. 
a

ELISA

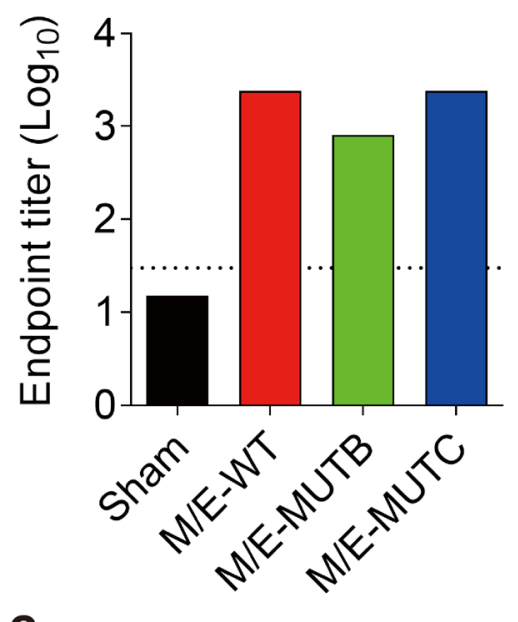

C

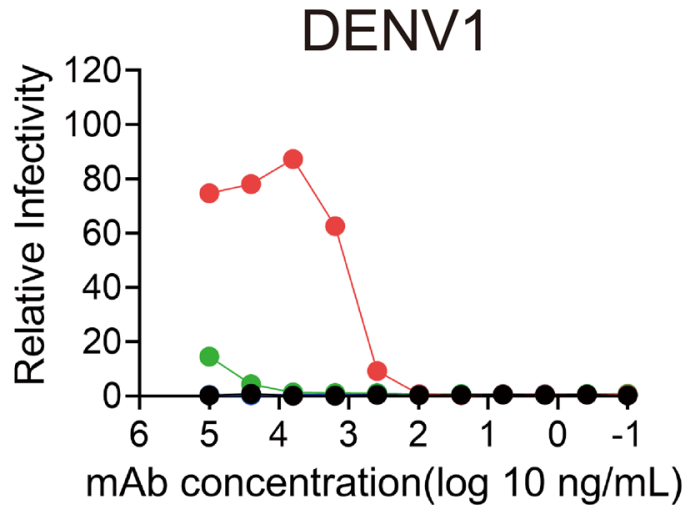

b
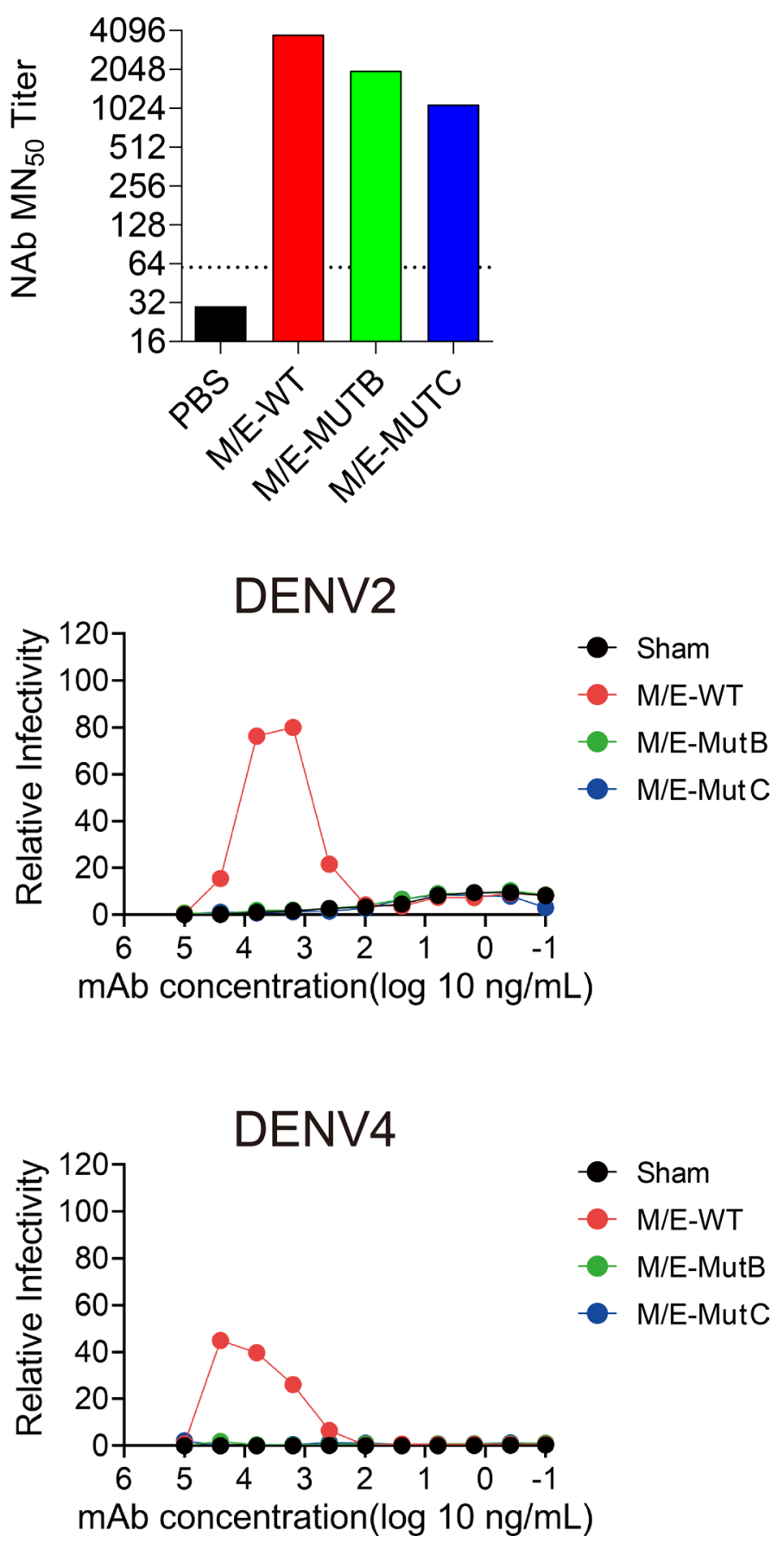

Extended Data Fig. 7 | Evaluation of the sera from donor mice, related to Figs. 3f,g. a,b, The pooled serum from donors were tested for ZIKV-E-specific $\operatorname{lgG}$ (a) and NAb titers (b). The dashed line indicates the LOD. c, ADE activities of the pooled donor sera in cell culture. Shown are the enhancement curves of K562 cells infected with DENV1, DENV2, DENV3, and DENV4, respectively, in the presence of serially diluted immunized mice sera as indicated. Infected cells were quantified by flow cytometry. Relative infectivity for each sample were normalized to the peak infectivity of mAb Z6. 

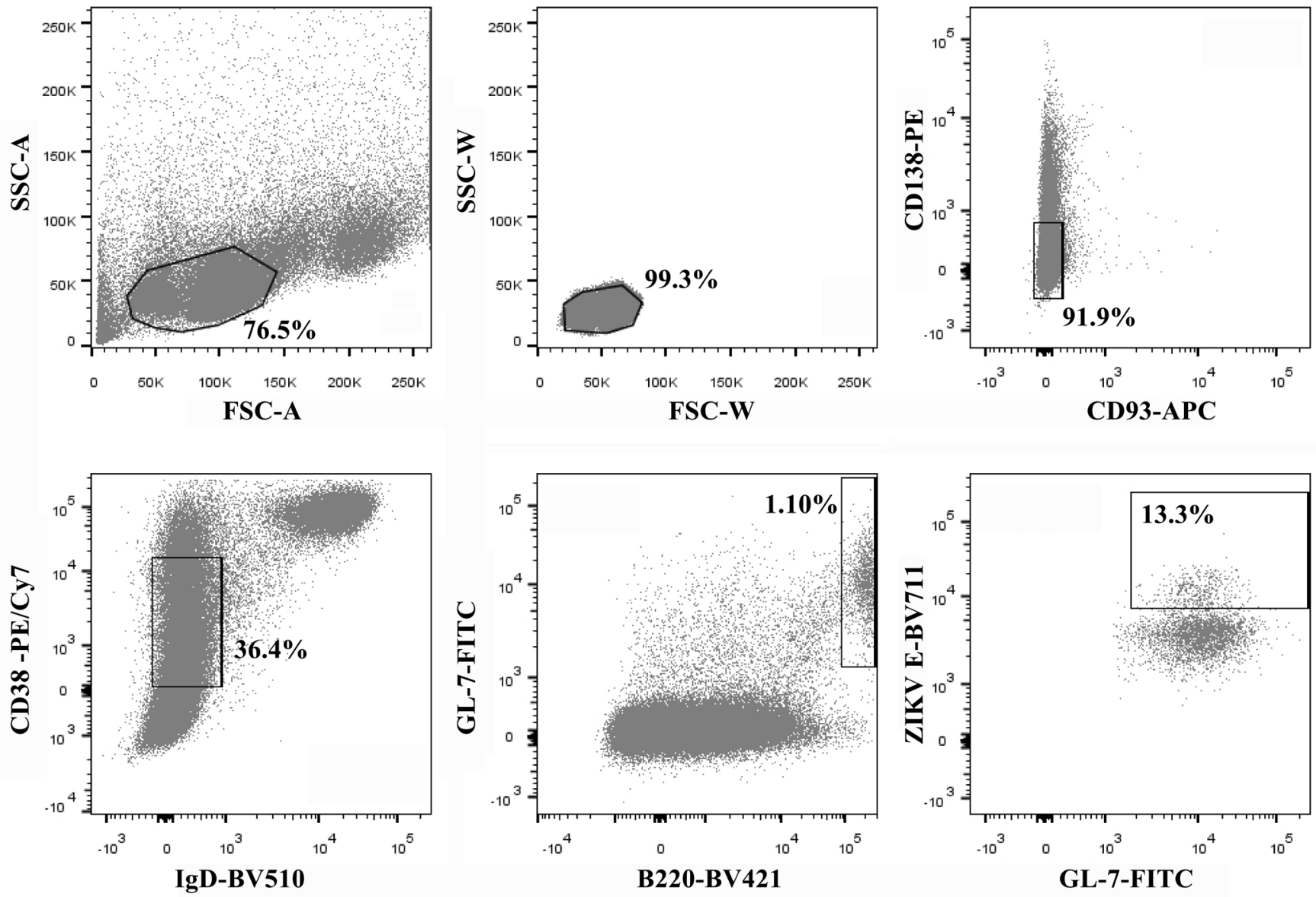

Extended Data Fig. 8 | FACS plots showing the gating strategy to isolate single ZIKV-E+ $B_{G C}$. The ZIKV-E-binding $B_{G C}\left(G L-7^{+} B 22 O^{\text {hi }} C D 38^{l o}\right.$ IgD-CD93-CD138-) from the lymph nodes of a group of BALB/C mice primed with ZIKV vaccines was sorted by flow cytometry. 
a

\section{Heavy Chain}

IGHV9-2-1*01/IGHJ4*01 ZWT. 1

ZWT. 2

ZWT. 3

$6 \mathrm{~B} 6 \mathrm{C}-1$

b

Light Chain

IGKV10-96*01/IGKJ1*01 ZWT. 1

ZWT. 2

ZWT. 3

$6 \mathrm{~B} 6 \mathrm{C}-1$

C

Heavy Chain

IGHV1-22*01/IGHJ2*01 IGHV1-22*01/IGH $4 * 01$

ZWT. 4

ZWT. 5

ZWT. 6

ZWT.6

2A10G6

d

Light Chain

IGKV14-111*01/IGKJ1*01 ZWT. 4

ZWT. 5

4G2

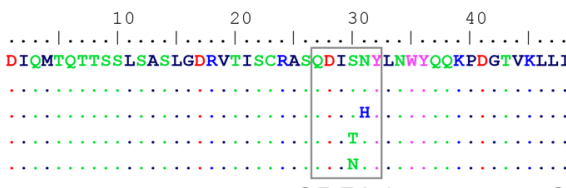

CDRL1

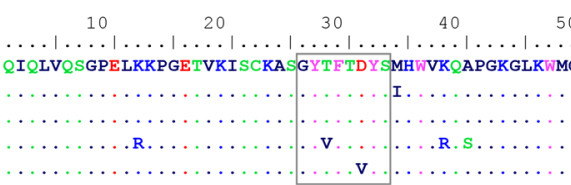

CDRH1

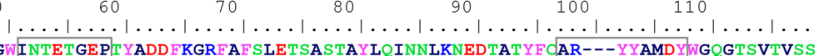

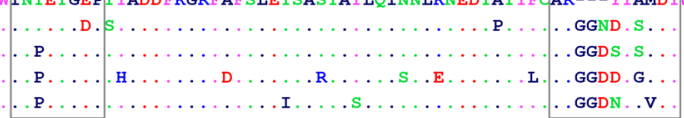

$\mathrm{CDRH} 2$

CDRH3

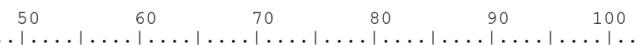

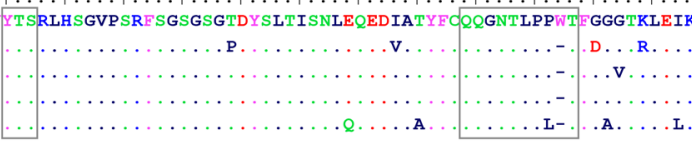

CDRL2

CDRL3
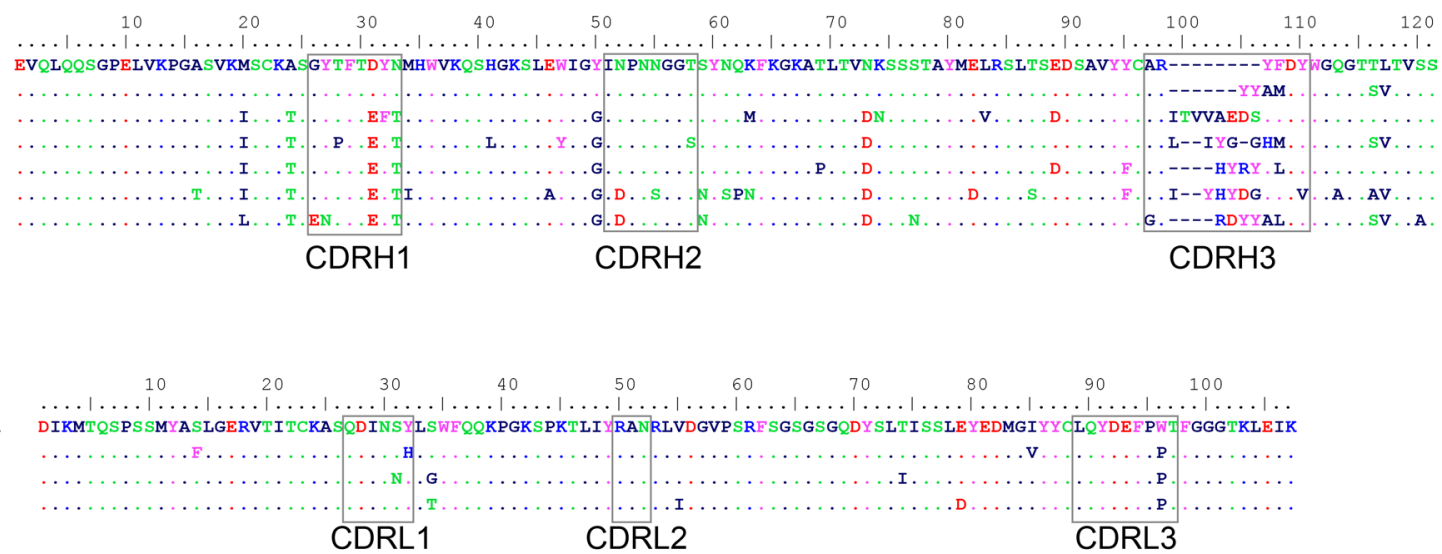

e

Light Chain

IGKV6 $-23 * 01 /$ IGKJ $2 * 01$ I GKV6-13*01/IGKJ2*01 ZWT. 6

2A10G6

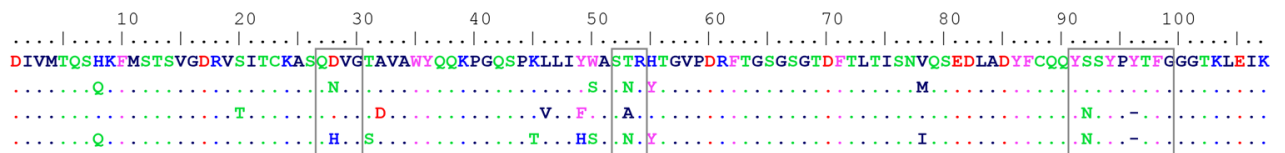

CDRL1
CDRL2
CDRL3

\begin{tabular}{ll}
\multicolumn{2}{c}{ Chemical Characteristics Amino Acids } \\
\hline Polar Positive charge & HKR \\
Polar Negative charge & DE \\
Polar Neutral & CNQST \\
Non-polar Neutral & AGILMPV \\
Non-polar Aromatic Neural & FWY
\end{tabular}

Extended Data Fig. 9 | Amino acid sequences and alignment of FLE mAbs. a, Amino acid sequence alignment of VH-region for mAb ZWT.1-3 and 6B6C-1. b. Amino acid sequence alignment of VL-region for mAb ZWT.1-3 and 6B6C-1. c, Amino acid sequence alignment of VH-region for mAb ZWT.4-6, 4G2 and 2A10G6. d, Amino acid sequence alignment of VL-region for mAb ZWT.4-5 and 4G2. e, Amino acid sequence alignment of VL-region for mAb ZWT.6 and 2A10G6. 
a

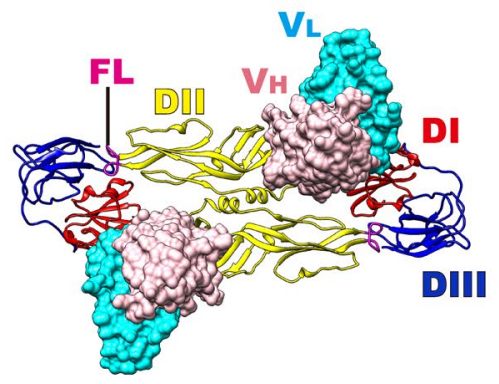

sE_MutC/Z3L1_scFv

b

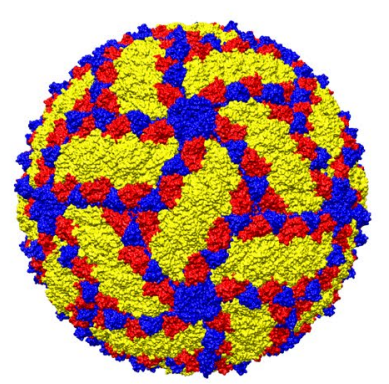

ZIKV particle

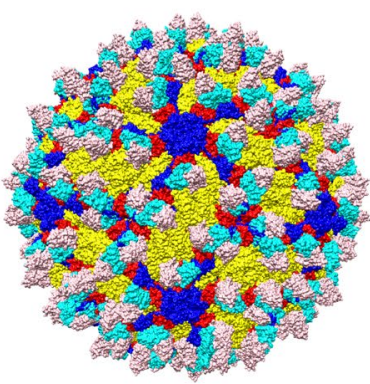

E-MutC/Z3L1 onto particle

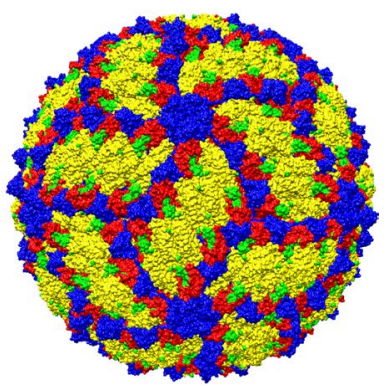

E-MutC onto particle

Extended Data Fig. 10 | Overview of E-MutC bound to Z3L1_scFv and model on virus particle. a, Overall structure of complex of sE-MutC/Z3L1_scFv. The sE-MutC is shown as ribbon, Z3L1_scFvs are showed as surface. $\mathbf{b}$, Left: the cryo-electron microscopy structure of the mature ZIKV particle (PDB: 5IZ7). Middle: ZIKV-E-MutC/Z3L1-scFv docked onto the the mature ZIKV particle (PDB: 5IZ7), Right: ZIKV-E-MutC docked onto the the mature ZIKV particle (PDB: 5IZ7). The Z3L1 footprint are highlighted in green. 


\section{Reporting Summary}

Nature Research wishes to improve the reproducibility of the work that we publish. This form provides structure for consistency and transparency in reporting. For further information on Nature Research policies, see our Editorial Policies and the Editorial Policy Checklist.

\section{Statistics}

For all statistical analyses, confirm that the following items are present in the figure legend, table legend, main text, or Methods section.

$\mathrm{n} / \mathrm{a}$ Confirmed

$\bigotimes$ The exact sample size $(n)$ for each experimental group/condition, given as a discrete number and unit of measurement

$\square$ \ A statement on whether measurements were taken from distinct samples or whether the same sample was measured repeatedly

The statistical test(s) used AND whether they are one- or two-sided

Only common tests should be described solely by name; describe more complex techniques in the Methods section.

$\bigotimes \square$ A description of all covariates tested

Х $\square$ A description of any assumptions or corrections, such as tests of normality and adjustment for multiple comparisons

$\triangle$ A full description of the statistical parameters including central tendency (e.g. means) or other basic estimates (e.g. regression coefficient) AND variation (e.g. standard deviation) or associated estimates of uncertainty (e.g. confidence intervals)

For null hypothesis testing, the test statistic (e.g. $F, t, r$ ) with confidence intervals, effect sizes, degrees of freedom and $P$ value noted Give $P$ values as exact values whenever suitable.

Х $\square$ For Bayesian analysis, information on the choice of priors and Markov chain Monte Carlo settings

Х $\square$ For hierarchical and complex designs, identification of the appropriate level for tests and full reporting of outcomes

$\triangle \square$ Estimates of effect sizes (e.g. Cohen's $d$, Pearson's $r$ ), indicating how they were calculated

\section{Our web collection on statistics for biologists contains articles on many of the points above.}

\section{Software and code}

Policy information about availability of computer code

Data collection Flow cytometry data were collected using BD FACSAria II flow cytometer or FACSCanton flow cytometer; qPCR data were collected by CFX96 Touch real-time PCR detection system (Bio-Rad, USA); ELISA data were collected by microplate reader (PerkinElmer, USA);SPR binding experiments were carried out using a BIAcore 8000 device (GE Healthcare); Libraries were sequenced by an Illumina NovaSeq (IIlumina, San Diego, CA)

Data analysis Flowjo software v.7.6.1 (Treestar Inc); GraphPad Prism version v6 \& v9.0.1; OriginPro 2018c (OriginLab Corp.); PyMOL version 2.5.0a0; UCSF Chimera version 1.15; BIAevaluation Version 4.1; The Cell Ranger Single-Cell Software Suite (versions 3.1.0); LoupeTM V(D)J Browser v3.0.0; HKL2000 version 1.74; CCP4 program suite v7.0; Coot version 0.94; IgBLAST v1.6.1; Illumina bcl2fastq 2.20; Phaser; Phenix. Refine; MolProbity;

For manuscripts utilizing custom algorithms or software that are central to the research but not yet described in published literature, software must be made available to editors and

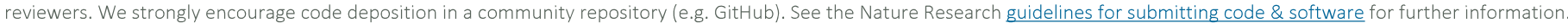

\section{Data}

Policy information about availability of data

All manuscripts must include a data availability statement. This statement should provide the following information, where applicable:

- Accession codes, unique identifiers, or web links for publicly available datasets

- A list of figures that have associated raw data

- A description of any restrictions on data availability

The crystal structures of ZIKV sE-Z6 and ZIKV sE-MutC-Z3L1 have been deposited in the Protein Data Bank (PDB) under accession codes 7BQ5 and 7BPK, respectively. The raw data for scBCR-seq have been deposited in https://www.scidb.cn/en/datalist?tag=1. Source data are provided with this paper. All other data supporting the 


\section{Field-specific reporting}

Please select the one below that is the best fit for your research. If you are not sure, read the appropriate sections before making your selection. $\bigotimes$ Life sciences $\quad \square$ Behavioural \& social sciences $\quad \square$ Ecological, evolutionary \& environmental sciences

For a reference copy of the document with all sections, see nature.com/documents/nr-reporting-summary-flat.pdf

\section{Life sciences study design}

All studies must disclose on these points even when the disclosure is negative.

Sample size No statistical test was used to determine the number of samples. Sample sizes were determined as appropriate to evaluate detection of large vaccine effects based on several studies done by us and others.

Data exclusions No data were excluded from any of the analysis.

Replication All the antibody assays were performed from each samples at least twice and the data were reproducible. All the other assays were performed once and all the biological replicates are presented.

Randomization Animals were randomly allocated to each group such that the age and weight at the time of allocation was normalized across group. For experiments not involving animals (Fig 1e,1f, 6a-i, 7a, Extended Data Fig.3, Extended Data Fig.5), randomization was not necessary as various samples were recored and analyzed by a computer software for extracting the significant differences. For structural study, randomization is not relevant.

Blinding

Blinding was not completed as assays were completed by the same team that immunized animals.

\section{Reporting for specific materials, systems and methods}

We require information from authors about some types of materials, experimental systems and methods used in many studies. Here, indicate whether each material, system or method listed is relevant to your study. If you are not sure if a list item applies to your research, read the appropriate section before selecting a response.

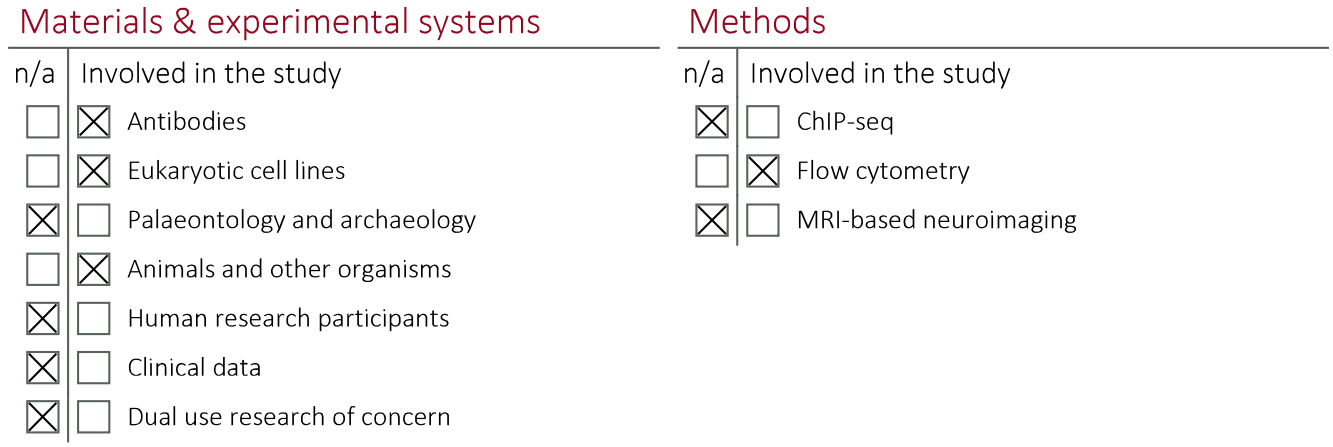

\section{Antibodies}

Antibodies used

GL-7-FITC, 553666, BD Biosciences, Clone GL7, PMID: 9323211, 1:100

CD138-PE, 553714, BD Biosciences, Clone 281-2, PMID: 11466358, 1:50

CD38-PE/Cy7, 102718, BioLegend, Clone 90, PMID: 31866068, 1:50

CD93-APC, 136510, BioLegend, Clone AA4.1, PMID: 31775041, 1:50

B220-BV421, 103240, BioLegend, Clone RA3-6B2, PMID: 24039898, 1:12.5

IgD-BV510, 563110, BD Biosciences, Clone 11-26c.2a, PMID: 7514440, 1:100

goat anti-mouse IgG-HRP, ab6789, Abcam, PMID: 30595451, 1:2000

Z3L1-HRP, produced in this paper; $1 \mathrm{ug} / \mathrm{ml}$ as a working concentration

The following monoclonal antibodies were generated in the current study. The concentration used in each experiment has been described in figures, legends or source data.

Z6, This paper;

2A10G6, This paper;

Z3L1, This paper;

Z23, This paper;

Z20, This paper;

ZWT.1, This paper; 
ZWT.2, This paper;

ZWT.3, This paper;

ZWT.4, This paper;

ZWT.5, This paper;

ZWT.6, This paper;

ZWT.7, This paper;

ZWT.8, This paper;

ZWT.9, This paper;

ZWT.10, This paper;

ZMutB.1, This paper;

ZMutB.2, This paper;

ZMutB.3, This paper;

ZMutB.4, This paper;

ZMutB.5, This paper;

ZMutB.6, This paper;

ZMutB.7, This paper;

ZMutB.8, This paper;

ZMutC.1, This paper;

ZMutC.2, This paper;

ZMutC.3, This paper;

ZMutC.4, This paper;

ZMutC.5, This paper;

ZMutC.6, This paper;

ZMutC.7, This paper;

ZMutC.8, This paper;

ZMutC.9, This paper;

ZMutC.10, This paper;

ZMutC.11, This paper;

ZMutC.12, This paper;

ZMutC.13, This paper;

Validation
All commercial available antibodies were validated by their source company as follows:
Flow cytometry:
-FITC anti-GL-7 (553666, BD Biosciences): same used in PMID: 33125897
-PE anti-CD138 (553714, BD Biosciences): same used in PMID: 29478774
-PE/Cy7 anti-CD38 (102718, BioLegend): same used in PMID: 28841417
-APC anti-CD93 (136510, BioLegend): same used in PMID: 30332630
-BV421 anti-B220 (103240, BioLegend): same used in PMID: 30726742
-BV510 anti-IgD (563110, BD Biosciences): same used in PMID: 31104946

\section{Eukaryotic cell lines}

Policy information about cell lines

Cell line source(s)

BHK21 cells (ATCC CCL-10); HEK293 cells (ATCC CRL-1573); human embryonic kidney cells 293T (HEK293T, ATCC CRL-3216); African green monkey kidney epithelial cells (Vero cells; ATCC CCL-81); K562 cells (ATCC CCL-243); C6/36 cells (ATCC, CRL-166)

Authentication

None of the cell lines were autenticated.

Mycoplasma contamination

All cell lines were tested negative for mycoplasma.

Commonly misidentified lines (See $\underline{\text { ICLAC }}$ register)

No commonly misidentified cell line was used in the study.

\section{Animals and other organisms}

Policy information about studies involving animals; ARRIVE guidelines recommended for reporting animal research

Laboratory animals

BALB/c mice (purchased from Beijing Vital River Laboratory Animal Technology Co., Ltd), 6-8-week-old, female; Ifnar1-/- mice (were purchased from the Institute of Laboratory Animal Science, Chinese Academy of Medical Sciences and Peking Union Medical College (CAMS\&PUMC), 5-6-week-old, both male and female; 5-week-old AG6 mice (deficient in both IFNAR1 and IFNGR) were generated from C57BL/6 background ifngr1-/- mice (Jackson Laboratories,\#003288) and ifnar1-/- A129 mice (B\&H company).

Wild animals

Study did not involve wild animals

Field-collected samples

Study did not involve samples collected from the field

Ethics oversight

All animal experiments were approved by the Committee on the Ethics of Animal Experiments of the IMCAS, and conducted in compliance with the recommendations in the Guide for the Care and Use of Laboratory Animals of the IMCAS Ethics Committee. 
Plots

Confirm that:

$\bigotimes$ The axis labels state the marker and fluorochrome used (e.g. CD4-FITC).

\The axis scales are clearly visible. Include numbers along axes only for bottom left plot of group (a 'group' is an analysis of identical markers).

\All plots are contour plots with outliers or pseudocolor plots.

$\bigotimes$ A numerical value for number of cells or percentage (with statistics) is provided.

\section{Methodology}

Sample preparation

Instrument

Software

Cell population abundance

Gating strategy

\Tick
HEK293T cells were transfected with pCAGGS plasmids encoding wild-type or mutated ZIKV-M/E proteins, or infected with AdC7 viruses encoding wild-type or mutated ZIKV-M/E proteins. After $48 \mathrm{hr}$, the cells were digested by trypsin and collected, followed by incubation in Fixation and Permeabilization solution buffer (BD, 554722) for $20 \mathrm{~min}$ at $4^{\circ} \mathrm{C}$. Then the cells were averagely divided into several parts and each part was stained with each mAb (Z6, 2A10G6, Z3L1, Z23 or Z20) for $1 \mathrm{hr}$ at $4^{\circ} \mathrm{C}$. The cells were then washed twice with $1 \times$ Perm/Wash buffer and stained with Goat Anti-Human IgG-FITC (Proteintech, USA) for $1 \mathrm{~h}$ at $4^{\circ} \mathrm{C}$. After wash twice with $1 \times$ Perm/Wash buffer, the cells were re-suspended in PBS. Subsequently, FITC-positive HEK293T cells were detected by FACSCanton flow cytometer (BD Bioscience, USA).

Single-cell suspensions were obtained from the lymph nodes of immunized mice. The cells from each group of mice were pooled together. Antibodies used for staining were anti-mouse GL-7-FITC, CD138-PE, CD38-PE/Cy7, CD93-APC, B220-BV421 and IgD-BV510. All antibodies were used according to manufactures' instructions. To stain the ZIKV-E-binding B cells, ZIKV-sE monomer (described above) and dimer (a kind gift from Rui Gong, Wuhan Institute of Virology, CAS, China) 64 were used as baits by biotinylation via Biotin-Protein Ligase/BirA Enzyme (GeneCopoeiaTM). BV711-Streptavidin was used to probe the biotinylated-ZIKV-E proteins. Antigen-specific BGC gated as GL-7+, B22Ohi, CD38lo, IgD-, CD93-, CD138- and ZIKV-E+ were sorted by BD FACSAria II flow cytometer (BD Biosciences).

Flow cytometry data were collected using BD FACSAria II flow cytometer or FACSCanton flow cytometer

Flowjo software v.7.6.1 (Treestar Inc)

Cell sorting was used for single-cell V(D)J RNA sequencing. By living cell counting, about $70 \%$ are living cell in WT-group, $80 \%$ in MutB-group and 74\% in MutC group. After sequencing, 490 cells have Ig sequences in WT-group, 321 cells have Ig sequences in MutB group and 621 cells have Ig sequences in MutC group. The details have been described in Fig. $4 \mathrm{~b}$

Cells were selected based on FSC-A vs.SSC-A; Singlets were selected using FSC-W vs. SSC-W; Terminally differential normal plasma cells were selected as CD138-PE+, CD93-APC-; Mature B cells were selected as CD38-PE/Cy7+, IgD-BV510-; GC B cells were selected as B220-BV421+, GL-7-FITC+; ZIKV E reactive B cells were selected by ZIKV E-BV711+

Tick this box to confirm that a figure exemplifying the gating strategy is provided in the Supplementary Information. 